\title{
Completeness for First-order Properties on Sparse Structures with Algorithmic Applications
}

\author{
JIAWEI GAO and RUSSELL IMPAGLIAZZO, University of California, San Diego \\ ANTONINA KOLOKOLOVA, Memorial University of Newfoundland \\ RYAN WILLIAMS, MIT
}

Properties definable in first-order logic are algorithmically interesting for both theoretical and pragmatic reasons. Many of the most studied algorithmic problems, such as Hitting Set and Orthogonal Vectors, are first-order, and the first-order properties naturally arise as relational database queries. A relatively straightforward algorithm for evaluating a property with $k+1$ quantifiers takes time $O\left(m^{k}\right)$ and, assuming the Strong Exponential Time Hypothesis (SETH), some such properties require $O\left(m^{k-\epsilon}\right)$ time for any $\epsilon>0$. (Here, $m$ represents the size of the input structure, i.e., the number of tuples in all relations.)

We give algorithms for every first-order property that improves this upper bound to $m^{k} / 2^{\Theta(\sqrt{\log n})}$, i.e., an improvement by a factor more than any poly-log, but less than the polynomial required to refute SETH. Moreover, we show that further improvement is equivalent to improving algorithms for sparse instances of the well-studied Orthogonal Vectors problem. Surprisingly, both results are obtained by showing completeness of the Sparse Orthogonal Vectors problem for the class of first-order properties under fine-grained reductions. To obtain improved algorithms, we apply the fast Orthogonal Vectors algorithm of References $[3,16]$.

While fine-grained reductions (reductions that closely preserve the conjectured complexities of problems) have been used to relate the hardness of disparate specific problems both within P and beyond, this is the first such completeness result for a standard complexity class.

CCS Concepts: • Theory of computation $\rightarrow$ Computational complexity and cryptography; Problems, reductions and completeness;

Additional Key Words and Phrases: Fine-grained complexity, first-order model checking, orthogonal vectors

ACM Reference format:

Jiawei Gao, Russell Impagliazzo, Antonina Kolokolova, and Ryan Williams. 2018. Completeness for Firstorder Properties on Sparse Structures with Algorithmic Applications. ACM Trans. Algorithms 15, 2, Article 23 (December 2018), 35 pages.

https://doi.org/10.1145/3196275

This research is supported by NSF grant CCF-1213151 from the Division of Computing and Communication Foundations (first two authors) and NSF grant CCF-1552651 (CAREER), last author. Any opinions, findings and conclusions or recommendations expressed in this material are those of the authors and do not necessarily reflect the views of the National Science Foundation.

The third author was partially supported by an NSERC Discovery grant. Some of the work was done while visiting UCSD. This work was done in part while the authors were visiting the Simons Institute for the Theory of Computing.

Authors' addresses: J. Gao and R. Impagliazzo, Department of Computer Science and Engineering, University of California, San Diego, 9500 Gilman Drive, Mail Code 0404, La Jolla, CA 92093-0404; emails: jiawei@ucsd.edu, russell@cs.ucsd.edu; A. Kolokolova, Department of Computer Science, Memorial University of Newfoundland, St. John's, NL A1B 3X5, Canada; email: kol@mun.ca; R. Williams, MIT CSAIL, 32 Vassar St, Cambridge, MA 02139; email: rrw@mit.edu.

Permission to make digital or hard copies of all or part of this work for personal or classroom use is granted without fee provided that copies are not made or distributed for profit or commercial advantage and that copies bear this notice and the full citation on the first page. Copyrights for components of this work owned by others than the author(s) must be honored. Abstracting with credit is permitted. To copy otherwise, or republish, to post on servers or to redistribute to lists, requires prior specific permission and/or a fee. Request permissions from permissions@acm.org.

(C) 2018 Copyright held by the owner/author(s). Publication rights licensed to ACM

1549-6325/2018/12-ART23 \$15.00

https://doi.org/10.1145/3196275 


\section{INTRODUCTION}

Fine-grained complexity aims to make complexity theory more relevant to algorithm design (and vice versa) by giving reductions that better preserve the times required for solving problems, and connecting algorithmic progress with complexity theory. While some of the key ideas can be traced back to parameterized algorithms and complexity [18, 20], studies of the exact complexity of NPcomplete problems [22-24, 29], and algorithmic consequences of circuit lower bounds [6, 8, 21, 25, $26,28,37]$, the full power of this approach has emerged only recently. This approach has given us new circuit lower bounds [32,34], surprising algorithmic improvements using circuit lower bound techniques $[3,15,16,31]$, and many new insights into the relative difficulty of substantially improving known algorithms for a variety of problems both within and beyond polynomial time.

One of the strengths of this approach also makes it seemingly more complicated. Fine-grained reductions often cut across traditional complexity hierarchies; for example, many results use a now-standard reduction from the NP-complete SAT problem down to the first-order definable (a.k.a. uniform $\mathrm{AC}^{0}$ ) orthogonal vectors problem. (Counterintuitively, this reduces a very hard problem to a problem in an extremely simple complexity class). However, different complete problems for the same complexity class can have different time complexities, meaning there may not be fine-grained reductions between them (or at least, that such reductions can be highly nontrivial.) Thus far, fine-grained complexity has remained focused on specific problems, rather than organizing problems into classes as in traditional complexity. As the field has grown, many fundamental relationships between problems have been discovered, making the graph of known results a somewhat tangled web of reductions $[1,2,5,9,11-13,27,36]$.

Here, we give the first results in fine-grained complexity that apply to an entire complexity class, namely the class of first-order definable properties (the uniform version of $A C^{0}$ ). This class is both algorithmically natural in that it contains many standard problems considered before (such as Hitting Set and Orthogonal Vectors), and motivated by its importance in logic and database theory. It is not difficult to see that checking whether a property expressible by a first-order formula with $k+1$ quantifiers holds on a given structure with $m$ records can be done in $O\left(m^{k}\right)$ time, and if Strong Exponential Time Hypothesis (SETH) is true, there are such properties that require $m^{k-o(1)}$ time to decide. ${ }^{1}$ For $k=1$, this is linear time and so cannot be improved. For each such problem with $k \geq 2$, we give an algorithm that solves it in $m^{k} / 2^{\Theta(\sqrt{\log m})}$ time. (This improves the standard algorithm by a factor more than any poly-log, but less than the polynomial improvement needed to refute SETH.) Moreover, we show that any further improvement is equivalent to a similar algorithmic improvement for the well-studied Orthogonal Vectors problem. Surprisingly, both results are obtained by showing that (a version of) the Orthogonal Vectors problem is complete under finegrained reductions for the class of all first-order properties. This is the first completeness result for a previously studied complexity class under fine-grained reducibility. To obtain the algorithmic results, we then apply the counter-intuitive algorithm for the Orthogonal Vectors problem of [3, 16], which uses techniques from circuit lower bounds.

In addition to introducing new algorithms and giving completeness results, our results clarify and simplify our understanding of "complexity within P." For many of the known SETH-hard problems of interest such as Edit Distance [9], Longest Common Subsequence [1, 5, 13], Dynamic Time Warping [1,13], Fréchet Distance [12], Succinct Stable Matching [27], and so on, the reduction from SAT passes through the Orthogonal Vectors problem. Thus, if any of these SETH-hard problems

\footnotetext{
${ }^{1}$ Informally, SETH is a hypothesis that CNF SAT cannot be solved substantially faster than $2^{n}$ time; see the Preliminaries for a formal statement.
} 

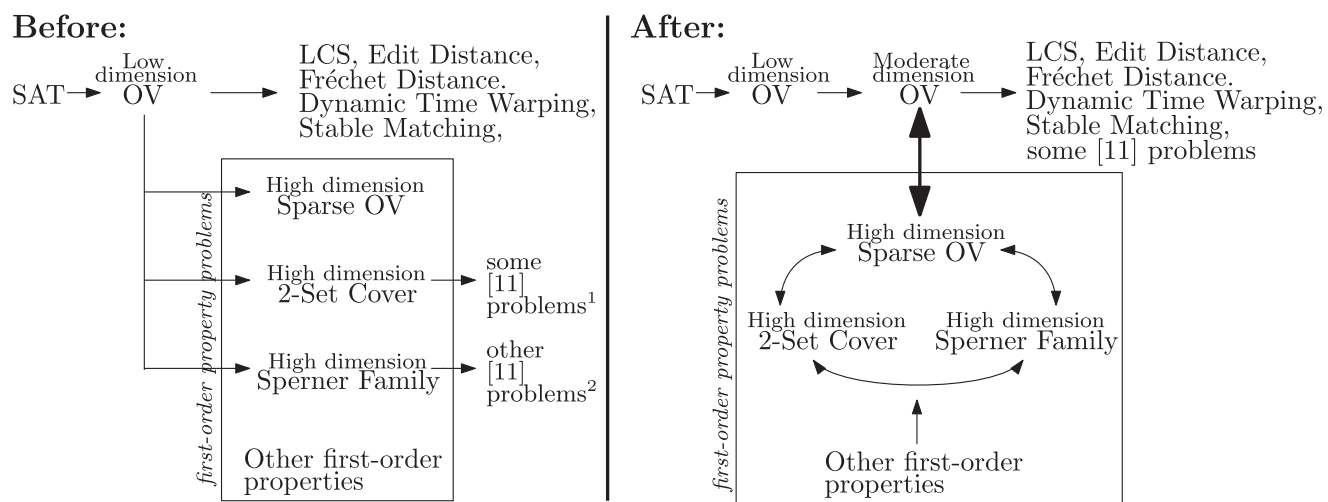

1 includes 3-Dominating Set and Bipartite Subset 2-Dominating Set.

2 includes Graph Dominated Vertex, Maximum Simple Family of Sets, and Subset Graph.

Fig. 1. A diagram of reductions. We simplify this picture and make the reductions to Edit Distance, LCS, and so on, more meaningful.

had substantially improved algorithms, all first-order properties would have similarly improved algorithms. Thus FOPC, the hypothesis that some first-order property does not have a substantially faster algorithm, is a useful intermediary between SETH and many of its consequences. FOPC is both equivalent to conjectures concerning many of the previously studied problems [11], and potentially more plausible to SETH-skeptics since it concerns an entire complexity class, while having most of the consequences of SETH. This is summarized in Figure 1. (See Section 2.3 for definitions of problems.)

While we concentrate on the general picture of complexity classes, even special cases of our results for specific problems are of interest. There were no similarly improved algorithms for Orthogonal Vectors with small total Hamming weight (Sparse OV) or related problems such as Sperner Family and 2-Set Cover (in the sparse high-dimensional case), and it was not previously known that the sparse versions of these problems were equivalent.

In addition to having a natural and useful complete problem, the class of first-order properties is important in itself. This class includes many problems studied in the fine-grained complexity literature such as Hitting Set, Orthogonal Vectors, Sperner Family, Diameter 2, Radius 2, $k$-Independent Set, $k$-Dominating Set, and so on. First-order properties are also extensively studied in complexity, logic (especially finite model theory and theory of databases) and combinatorics. Algorithms for model-checking first-order properties are inherent in databases (the core of the relational database language $S Q L$ is equivalent to first-order properties). Roughly speaking, first-order properties are essentially the uniform version of $\mathrm{AC}^{0}$ in the complexity literature [10].

Since fine-grained complexity is concerned with exact time complexities (distinguishing, e.g., $n^{1.9}$ time from $n^{2}$ time), the problem representation is significant. For graph problems, there are two standard representations: adjacency lists (which are good for sparse graphs), in which running time is analyzed with respect to the number of edges $m$, and adjacency matrices (good for dense graphs), in which the runtime is a function of the number of vertices, $n$. For several reasons, we use the sparse adjacency list (list of tuples) representation. First, many of the problems considered such as Orthogonal Vectors are hard already on sparse instances. Second, the complexity of first-order problems in the dense model is somewhat unclear, at least for numbers of quantifiers between 3 and 7 [33]. Third, the sparse model is more relevant for first-order model checking, as databases are represented as lists of records. 


\subsection{First-order Properties}

The problem of deciding whether a structure satisfies a logical formula is called the model checking problem. In relational databases, first-order model checking plays an important role, as first-order queries capture the expressibility of relational algebra. In contrast to the combined complexity, where the database and query are both given as input, the data complexity measures the running time when the query is fixed. The combined complexity of first-order queries is PSPACE-complete, but the data complexity is in LOGSPACE [30]. Moreover, these problems are also major topics in parameterized complexity theory. In Reference [19], Flum and Grohe organize parameterized first-order model-checking problems (many of which are graph problems) into hierarchical classes based on their quantifier structures. Here, we study model checking from the fine-grained complexity perspective.

More specifically, let $\varphi$ be a fixed first-order sentence containing free predicates of arbitrary constant arity (and no other free variables). For example, the $k$-Orthogonal Vectors $(k-\mathrm{OV})$ problem can be expressed by a $(k+1)$-quantifier formula $\varphi=\left(\exists v_{1} \in A_{1}\right) \ldots\left(\exists v_{k} \in A_{k}\right)(\forall i)\left[\bigvee_{j=1}^{k} \neg\left(v_{j}[i]=\right.\right.$ 1)]. The model-checking problem for $\varphi$, denoted $M C_{\varphi}$, is deciding whether $\varphi$ is true on a given input structure interpreting predicates in $\varphi$ (e.g., given $k$ sets of vectors, decide $k$-OV). We sometimes refer to structures as "hypergraphs" ("graphs" when all relations are unary or binary), and relations as edges or hyperedges. We use $n$ to denote size of the universe of the structure and $m$ the total number of tuples in all its relations (size of the structure). Many graph properties such as $k$-clique have natural first-order representations, and set problems such as Hitting Set are representable in first-order logic using a relation $R(u, S) \equiv(u \in S)$.

We use notation $M C(\Phi)$ for a class of model-checking problems for $\varphi \in \Phi$, with the main focus on classes of $(k+1)$-quantifier $\varphi$ with $k \geq 1$ (denoted $M C(k+1)$ ) and restrictions of this class to specific quantifier prefixes (e.g., $M C(\exists \exists \forall)$ for 3-quantifier $\varphi$ with quantifier prefix $\exists \exists \forall$ when written in prenex normal form). For formal definitions and more examples see Sections 2.2 and 2.3 .

We propose the following conjecture on the hardness of model checking of first-order properties.

First-order property conjecture (FOPC): There is an integer $k \geq 2$, so that there is a $(k+1)$ quantifier first-order property that cannot be decided in $O\left(m^{k-\epsilon}\right)$ time, for any $\epsilon>0$.

\subsection{Orthogonal Vectors}

In the Orthogonal Vectors (OV) problem, we are given a set $A$ of $n$ Boolean vectors of dimension $d$, and must decide if there are $u, v \in A$ such that $u$ and $v$ are orthogonal, i.e., $u[i] \cdot v[i]=0$ for all indices $i \in\{1, \ldots, d\}$. Another (equivalent) version is to decide with two sets $A$ and $B$ of Boolean vectors whether there are $u \in A$ and $v \in B$ so that $u$ and $v$ are orthogonal. A naïve algorithm for OV runs in time $O\left(n^{2} d\right)$, and the best known algorithm runs in $n^{2-\Omega(1 / \log (d / \log n))}[3,16]$.

In this article, we introduce a version of OV we call the Sparse Orthogonal Vectors (Sparse OV) problem, where the input is a list of $m$ vector-index pairs $(v, i)$ for each $v[i]=1$ (corresponding to the adjacency list representation of graphs) and complexity is measured in terms of $m$; we usually consider $m=O\left(n^{1+\gamma}\right)$ for some $0 \leq \gamma<1$. The popular hardness conjectures on OV restrict the dimension $d$ to be between $\omega(\log n)$ (low dimension) and $n^{o(1)}$ (moderate dimension); however in Sparse OV we do not restrict $d$.

We thus identify three versions of Orthogonal Vector Conjecture, based on the size of the dimension $d$. In all three conjectures the complexity is measured in the word RAM model with $O(\log n)$ bit words.

Low-dimension OVC (LDOVC): For all $\epsilon>0$, there is no $O\left(n^{2-\epsilon}\right)$ time algorithm for OV with dimension $d=\omega(\log n)$. 
Moderate-dimension OVC (MDOVC): For all $\epsilon>0$, there is no $O\left(n^{2-\epsilon}\right.$ poly $\left.(d)\right)$ time algorithm that solves $\mathrm{OV}$ with dimension $d$.

Sparse OVC (SOVC): For all $\epsilon>0$, there is no $O\left(m^{2-\epsilon}\right)$ time algorithm for Sparse OV, where $m$ is the total Hamming weight of input vectors.

It is known that SETH implies LDOVC [31]. Because MDOVC is a weakening of LDOVC, it follows from the latter. ${ }^{2}$ Like LDOVC, MDOVC also implies the hardness of problems including Edit Distance, LCS, and so on. Here we further show that MDOVC and SOVC are equivalent (see Lemma 1.1).

OV can be extended to the $k$-OV problem for any integer $k \geq 2$ : given $k$ sets $A_{1}, \ldots, A_{k}$ of Boolean vectors, determine if there are $k$ different vectors $v_{1} \in A_{1}, \ldots, v_{k} \in A_{k}$ so that for all indices $i, \prod_{j=1}^{k} v_{j}[i]=0$ (that is, their inner product is 0 ). We naturally define a sparse version of $k$-OV similar to Sparse OV, where all ones in the vectors are given in a list.

\subsection{Main Results}

Completeness. First, we show that conjectures for OV defined on dense (moderate-dimension) and sparse models are equivalent under fine-grained reductions, which means MDOVC is true iff SOVC is true (see Lemma 6.2). This also holds for $k$-OV.

Lemma 1.1. For any integer $k \geq 2$, there exist $\delta, \epsilon>0$ and $a\left(n^{k-\epsilon}\right)$ time algorithm solving $k-O V$ with dimension $d=n^{\delta}$, if and only if there is an $\epsilon^{\prime}>0$ and a $O\left(m^{k-\epsilon^{\prime}}\right)$ time algorithm for Sparse $k-O V$, where $m$ is the total Hamming weight of all input vectors.

Our main result establishes an equivalence of MDOVC and FOPC, showing the completeness of Sparse OV and hardness of (dense) OV for the class of first-order property problems.

THEOREM 1.2. MDOVC, SOVC, and FOPC are equivalent.

This article also proves a hardness and completeness result for $k$-OV, connecting one combinatorial problem to a large and natural class of logical problems. The following theorem states that Sparse $k$-OV is complete for $M C\left(\exists^{k} \forall\right)$ (and its negation form $M C\left(\forall^{k} \exists\right)$ ), and hard for $M C\left(\forall \exists^{k-1} \forall\right)$ (and its negation form $M C\left(\exists \forall^{k-1} \exists\right)$ ) under fine-grained reductions.

THEOREM 1.3. If Sparse $k-O V$ with total Hamming weight $m$ can be solved in time $O\left(m^{k-\epsilon}\right)$ for some $\epsilon>0$, then all problems in $M C\left(\exists^{k} \forall\right), M C\left(\forall^{k} \exists\right), M C\left(\forall \exists^{k-1} \forall\right)$, and $M C\left(\exists \forall^{k-1} \exists\right)$ are solvable in time $O\left(m^{k-\epsilon^{\prime}}\right)$ for some $\epsilon^{\prime}>0$.

$M C\left(\exists^{k} \forall\right)$ and $M C\left(\forall^{k} \exists\right)$ are interesting sub-classes of $M C(k+1)$ : if Nondeterministic SETH is true, then all SETH-hard problems in $M C(k+1)$ are contained in $M C\left(\exists^{k} \forall\right)$ or $M C\left(\forall^{k} \exists\right)([14])$.

We will also show that the 2-Set Cover problem and the Sperner Family problem, both in $M C(\exists \exists \forall)$, are equivalent to Sparse OV under fine-grained reductions and thus complete for firstorder properties under fine-grained reductions.

Algorithmic results. Combining our reductions with the surprisingly fast algorithm for Orthogonal Vectors by References [3,16], we obtain improved algorithms for every problem representable as a $(k+1)$-quantifier first-order property.

THEOREM 1.4. There is an algorithm solving MC $(k+1)$ in time $m^{k} / 2^{\Theta(\sqrt{\log m})}$.

Let us consider the above results in context with prior work on the fine-grained complexity of first-order properties. In Reference [33], Ryan Williams studied the fine-grained complexity of

${ }^{2}$ Although dimension $d$ is not restricted, we call it "moderate dimension" because such an algorithm only improves on the naive algorithm if $d=n^{O(\epsilon)}$. 
dense instances of first-order graph properties. He gave an $n^{k+o(1)}$-time algorithm for $M C(k+1)$ on graphs when $k$ is a sufficiently large constant, and showed that $M C(k+1)$ requires at least $n^{k-o(1)}$ time under SETH. His algorithms only apply to graphs (they look difficult to generalize to even ternary relations), and it seems difficult to point to a specific simple complete problem in this setting. To compare, our results show that the sparse case of $M C(k+1)$ (for all c-ary relations, for all constants $c$ ) is captured by very simple problems (e.g., sparse Orthogonal Vectors), which also leads to an algorithmic improvement for all $c$-ary relations.

\subsection{Organization of This Paper}

Section 1 introduced the motivation, some definitions and statements of the main results. In Section 2, we give the formal definitions of the reductions we use, as well as detailed definitions of first-order properties, with first-order representations of common problems in fine-grained complexity. We present a general outline of the proofs in Section 3, and a high-level explanation of key techniques in Section 4.

The full proof starts with the reduction from $M C\left(\exists^{k} \forall\right)$ to $k$-OV (Section 5), with randomized universe-shrinking self-reduction described in Section 5.1, which is then derandomized in Section 6. Section 8 presents the reduction from $M C\left(\forall \exists^{k-1} \forall\right)$ to $k$-OV, and Section 10 discusses the $m^{k} / 2^{\Theta(\sqrt{\log m})}$ time algorithm for Sparse OV and, therefore, $M C(k+1)$. We conclude with open problems in Section 11.

\section{PRELIMINARIES}

\subsection{Reductions}

To establish the relationship between complexities of different problems, we use the notion of fine-grained reductions as defined in Reference [36]. These reductions establish conditional hardness results of the form "If one problem has substantially faster algorithms, then so does another problem." We will also use exact complexity reductions (see definition 2.2), which strengthen the above claim to "if one problem has algorithms improved by a factor $s(m)$, then another problem can be improved by a factor $s^{c}(m)$ " for some constant $c$. (Note that some fine-grained reductions already have this property.) The underlying computational model is the Word RAM with $O(\log n)$ bit words.

Definition 2.1 (Fine-grained reduction $\left.\left(\leq_{F G R}\right)\right)^{3}$. Assume that $L_{1}$ and $L_{2}$ are languages and $T_{1}$ and $T_{2}$ are their conjectured running time lower bounds, respectively, where constant factors may be omitted. Then we say $\left(L_{1}, T_{1}\right) \leq_{F G R}\left(L_{2}, T_{2}\right)$ if for every $\epsilon>0$, there exists $\epsilon^{\prime}>0$, and an algorithm $A_{L_{1}}$ for $L_{1}$ that runs in time $T_{1}(n)^{1-\epsilon^{\prime}}$ on inputs of length $n$, making $q$ calls to an oracle for $L_{2}$ with query lengths $n_{1}, \ldots, n_{q}$, where $\sum_{i=1}^{q}\left(T_{2}\left(n_{i}\right)\right)^{1-\epsilon} \leq\left(T_{1}(n)\right)^{1-\epsilon^{\prime}}$.

Thus, if $L_{2}$ has an algorithm substantially faster than $T_{2}, L_{1}$ can be solved substantially faster than $T_{1}{ }^{4}$

\footnotetext{
${ }^{3}$ To be more precise, this is the fine-grained Turing reduction. For the mapping reductions that preserve fine-grained complexity, we refer to them as fine-grained mapping reductions.

${ }^{4}$ In almost all fine-grained reductions, $T_{1} \geq T_{2}$, that is, we usually reduce from harder problems to easier problems, which may seem counter-intuitive. A harder problem $L_{1}$ can be reduced to a easier problem $L_{2}$ with $T_{1}>T_{2}$ in two ways: by making multiple calls to an algorithm solving $L_{2}$ and/or by blowing up the size of the $L_{2}$ instance (e.g., the reduction from CNFSAT to OV [31]). All reductions from higher complexity to lower complexity problems in this article belong to the first type.

Actually, it is harder to fine-grained reduce from a problem with lower time complexity to a problem with higher time complexity (e.g., prove that $\left.\left(M C(k), m^{k-1}\right) \leq_{F G R}\left(M C(k+1), m^{k}\right)\right)$, because this direction often needs creating instances with size much smaller than the original instance size.
} 
To simplify transferring algorithmic results, we define a stricter variant of fine-grained reductions, which we call exact reductions. These reductions satisfy a stronger reducibility notion.

Definition 2.2 (Exact complexity reduction $\left(\leq_{E C}\right)$ ). Let $L_{1}$ and $L_{2}$ be languages and let $T_{1}, T_{2}$ denote time bounds. Then $\left(L_{1}, T_{1}\right) \leq_{E C}\left(L_{2}, T_{2}\right)$ if there exists an algorithm $A_{L_{1}}$ for $L_{1}$ running in time $T_{1}(n)$ on inputs of length $n$, making calls to oracle of $L_{2}$ with query lengths $n_{1}, \ldots, n_{q}$, where $q$ is the number of calls and $\sum_{i=1}^{q} T_{2}\left(n_{i}\right) \leq T_{1}(n)$.

That is, if $L_{2}$ is solvable in time $T_{2}(n)$, then $A_{L_{1}}$ solves $L_{1}$ in time $T_{1}(n)$.

\subsection{Model Checking for First-order Logic}

Let $R_{1}, \ldots, R_{r}$ be predicates of constant arities $a_{1}, \ldots, a_{r}$ (a vocabulary). A finite structure over the vocabulary $R_{1}, \ldots, R_{r}$ consists of a universe $U$ of size $n$ together with $r$ lists, one for every $R_{i}$, of $m_{i}$ tuples of elements from $U$ on which $R_{i}$ holds. Let $m=\sum_{i=1}^{r} m_{i}$; viewing the structure as a database, $m$ is the total number of records in all tables (relations).

We loosely use the term hypergraph to denote an arbitrary structure; in this case, we refer to its universe as a set of vertices $V=\left\{v_{1}, \ldots, v_{n}\right\}$ and call tuples $\left(v_{1}, \ldots, v_{a_{i}}\right)$ such that $R_{i}\left(v_{1}, \ldots, v_{a_{i}}\right)$ holds hyperedges (labeled $R_{i}$ ). A set of all $R_{i}$-labeled hyperedges in a given hypergraph is denoted by $E_{R_{i}}$ or just $E_{i}$; the structure is denoted by $G=\left(V, E_{1}, \ldots, E_{r}\right)$. Similarly, we use the term graph for structures with only unary and binary relations (edges); here, we mean edge-labeled vertex-labeled directed graphs with possible self-loops, as we allow multiple binary and unary relations and relations do not have to be symmetric. This allows us to use graph terminology such as a degree (the number of (hyper)edges containing a given vertex) or a neighbourhood of a vertex.

Let $\varphi$ be a first-order sentence (i.e., formula without free first-order variables) containing predicates $R_{1}, \ldots, R_{r}$. Let $k$ be the number of quantifiers in $\varphi$. Without changing $k$, we can write $\varphi$ in prenex form. The model-checking problem for a first-order property $\varphi, M C_{\varphi}$, is: given a structure (hypergraph) $G$, determine whether $\varphi$ holds on $G$ (denoted by $G \mid=\varphi$ ). For a class of formulas $\Phi$, we use the notation $M C(\Phi)$ for a class of model-checking problems for $\varphi \in \Phi$, with shortcuts $M C(k)$ for $\Phi=k$-quantifier first-order formulas in prenex form, and $M C\left(Q_{1} \ldots Q_{k}\right)$ for first-order prenex formulas with quantifier prefix $Q_{1} \ldots Q_{k}$, with a shortcut $Q_{i}^{c}$ denoting $c$ consecutive occurrences of $Q$ (e.g., $\left.M C\left(\exists^{k} \forall\right)\right)$.

We assume that (hyper)graphs are given as a list of $m$ (hyper)edges, with each hyperedge encoded by listing its elements. In the Word RAM model with $O(\log n)$ bit words, the size of an encoding of a hypergraph is $O(n+m)$ words, and an algorithm can access a hyperedge in constant time. With additional $O(m)$ time preprocessing, we can compute degrees and lists of incident edges for each vertex, and store them in a hash table for a constant-time look-up; edges incident to a vertex can then be listed in time proportional to its degree. We also assume that $m \geq n$, with every vertex incident to some edge, because the interesting instances are in this case. Moreover, we assume the (hyper)graph is $k$-partite, where $k$ is the number of variables in $\varphi$, so that each variable is selected from a distinct vertex set. From any (hyper)graph, the construction of this $k$-partite graph needs a linear time, linear space blowup preprocessing that creates at most $k$ duplicates of the vertices and $k^{2}$ duplicates of the edges. Finally, we treat domains of quantifiers as disjoint sets forming a partition of the universe; any structure can be converted into this form with constant increase of the universe size. We also view predicates on different variable sets (e.g., $R\left(x_{1}, x_{2}\right)$ vs. $R\left(x_{2}, x_{4}\right)$ vs. $\left.R\left(x_{4}, x_{4}\right)\right)$ as different predicates, and partition corresponding edge sets appropriately.

The focus of this article is on sparse structures, that is, the case when $m \leq O\left(n^{1+\gamma}\right)$ for some $\gamma$ such that $0 \leq \gamma<1$. In particular, all $E_{i}$ are sparse relations; we use the term co-sparse to refer to complements of sparse relations. We will usually measure complexity as a function of $m$. From the 
following baseline algorithm that will be proved in Section 9.1, the sparse assumption is without loss of generality.

Claim 2.1 (Baseline Algorithm). $M C(k+1)$ is solvable in time $O\left(n^{k-1} m\right)$.

\subsection{Common Problems and Conjectures}

In the CNF-SAT problem, given a Boolean formula $F$ in CNF form (conjunction of disjunctions of (possibly negated) variables), the goal is to determine whether there is an assignment of Boolean values to variables of $F$ that makes $F$ true. In $k$-CNF-SAT, every clause (disjunction) can have at most $k$ literals. We refer to the following conjecture about complexity of solving CNF-SAT:

Strong Exponential Time Hypothesis (SETH) ${ }^{5}$ : For every $\epsilon>0$, there exists a $k \geq 2$ so that $k$-CNF-SAT cannot be solved in time $O\left(2^{n(1-\epsilon)}\right)$.

Below we list some problems studied in fine-grained complexity, with their first-order definitions on structures with unary and binary relations.

- Graph problems. The input structure is $G=(V, E)$ with a universe $V$ and a binary relation E.

1. Diameter-2: $\left(\forall x_{1}\right)\left(\forall x_{2}\right)\left(\exists x_{3}\right)\left[E\left(x_{1}, x_{3}\right) \wedge E\left(x_{3}, x_{2}\right)\right]$.

2. Radius-2: $\left(\exists x_{1}\right)\left(\forall x_{2}\right)\left(\exists x_{3}\right)\left[E\left(x_{1}, x_{3}\right) \wedge E\left(x_{3}, x_{2}\right)\right]$.

3. $k$-Clique: $\left(\exists x_{1}\right) \ldots\left(\exists x_{k}\right)\left[\bigwedge_{i, j \in\{1, \ldots, k\}, i \neq j} E\left(x_{i}, x_{j}\right)\right]$. More generally, for a fixed graph $H$ of $k$ vertices, deciding if $H$ is a subgraph or induced subgraph of the input graph $G$ (e.g., the $k$-Independent Set problem) can be expressed in a similar way.

4. $k$-Dominating Set: $\left(\exists x_{1}\right) \ldots\left(\exists x_{k}\right)\left(\forall x_{k+1}\right)\left[\bigvee_{i=1}^{k} E\left(x_{i}, x_{k+1}\right)\right]$.

- Set problems. The inputs are set families $\mathcal{S}$ or $\mathcal{S}_{1}, \ldots, \mathcal{S}_{k}$ over a universe $U$. Here, all sets are given explicitly and represented by first-order variables. These structures contain a single binary predicate $\in$.

1. Hitting Set: ${ }^{6}(\exists H \in \mathcal{S})(\forall S \in \mathcal{S})(\exists x)[(x \in H) \wedge(x \in S)]$.

2. k-Set Packing: $\left(\exists S_{1} \in \mathcal{S}\right) \ldots\left(\exists S_{k} \in \mathcal{S}\right)(\forall x)\left[\bigvee_{i=1}^{k}\left(\left(x \in S_{i}\right) \rightarrow \bigwedge_{j \neq i}\left(x \notin S_{j}\right)\right)\right]$.

3. $k$-Empty Intersection $(k-\mathrm{OV}):\left(\exists S_{1} \in \mathcal{S}_{1}\right) \ldots\left(\exists S_{k} \in \mathcal{S}_{k}\right)(\forall u \in U)\left[\bigvee_{i=1}^{k} \neg\left(u \in S_{i}\right)\right]$.

4. $k$-Set Cover: $\left(\exists S_{1} \in \mathcal{S}_{1}\right) \ldots\left(\exists S_{k} \in \mathcal{S}_{k}\right)(\forall u \in U)\left[\bigvee_{i=1}^{k}\left(u \in S_{i}\right)\right]$.

5. Set Containment: $\left(\exists S_{1} \in \mathcal{S}_{1}\right)\left(\exists S_{2} \in \mathcal{S}_{2}\right)(\forall u \in U)\left[\left(\neg\left(u \in S_{1}\right)\right) \vee\left(u \in S_{2}\right)\right]$.

$k$-Empty Intersection is equivalent to $k$-OV, and Set Containment is equivalent to Sperner Family problem. See Section 1.2 for definitions and conjectures for variants of the Orthogonal Vectors problem.

The Hitting Set Conjecture states that $\forall \epsilon>0$ there is no $O\left(n^{2-\epsilon}\right)$ time algorithm for the Hitting Set problem with set sizes bounded by $d=\omega(\log n)$. It implies LDOVC; subquadratic approximation algorithms for Diameter-2 and Radius- 2 would, respectively, refute the LDOVC and the Hitting Set Conjecture [4].

\section{OVERVIEW}

The main technical part of this article is in the proof of Theorem 1.3 showing hardness of $k$-OV for model-checking of $\exists^{k} \forall$ formulas under fine-grained reductions. The idea is to represent $\exists^{k} \forall$ formulas using combinations of basic " $k$-OV like" problems, each of which is either easy (solvable substantially faster than $m^{k}$ time for sparse instances) or can be fine-grained reduced to $k$-OV. The

\footnotetext{
${ }^{5}$ Some define SETH over randomized algorithms instead of deterministic ones.

${ }^{6}$ Other versions of Hitting Set, where the sets are not given explicitly are second-order logic problems. Our definition here is consistent with the version in the Hitting Set Conjecture.
} 


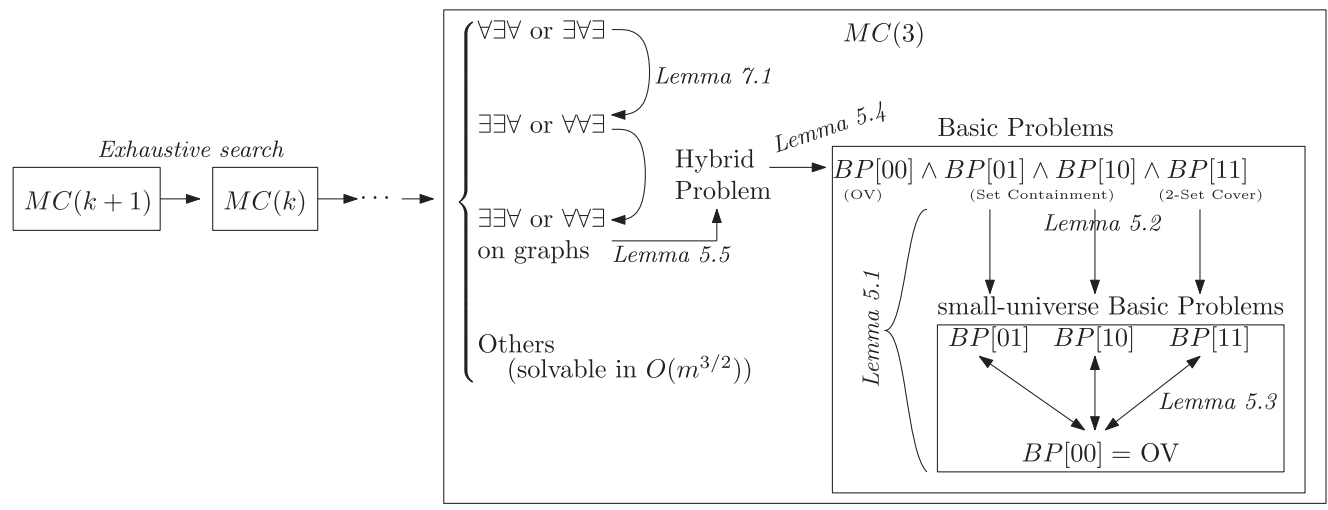

Fig. 2. Overview of the reduction process for Theorem 1.2.

latter is achieved using a universe-shrinking self-reduction, which converts a given instance of a basic problem to a denser instance on a smaller universe, thus reducing Sparse $k$-OV to $k$-OV with dimension $n^{\delta}$ and proving Lemma 1.1. Converting an $M C\left(\exists^{k} \forall\right)$ to the "hybrid problem" combining all $2^{k}$ basic problems is done for graphs (all relations have arity at most 2); however, we show that this is the hardest case. Additionally, $M C\left(\forall \exists^{k-1} \forall\right)$ is reduced to $M C\left(\exists^{k} \forall\right)$.

In Theorem 1.2 and Theorem 1.4, we consider the class of all $k+1$-quantifier first-order properties $M C(k+1)$, and reduce it to $2-\mathrm{OV}$, proceeding to use a faster algorithm for 2-OV to speed up model checking. The first step is to brute-force over first $k-2$ quantified variables, reducing to three-quantifier case at the cost $O\left(n^{k-2}\right)$. The quantifier prefix $\exists \exists \forall$, with 2-OV and other basic problems (to be defined in Section 5.1), is the hardest $\left(\exists^{3}, \forall \exists \exists\right.$ and their complements are easy, and the rest reduce to $\exists \exists \forall$ ). Appealing to lemmas in the proof of Theorem 1.3 with $k=2$ completes the proof of Theorem 1.2 (see Figure 2 for details), and applying the OV algorithm in References [3, 16] gives Theorem 1.4 .

\subsection{Reduction from $M C(k+1)$ to $\mathrm{OV}$}

The following outlines the reduction from any arbitrary problem in $M C(k+1)$ to OV for any integer $k \geq 2$, thus proving that FOPC implies SOVC. For the other direction of this eqiuvalence, SOVC implies FOPC because Sparse OV is in $M C(3)$. The equivalence between SOVC and MDOVC is proven in Lemma 1.1, which in turn follows from Lemma 5.2, Lemma 6.2, and corollary 5.3.

(1) With brute-force over tuples of first $k-2$ variables, we reduce from the $(k+1)$-quantifier problem $M C_{\varphi}$ down to a 3-quantifier problem $M C_{\varphi^{\prime}}$. Thus, improving the $O\left(m^{2}\right)$ algorithm for $M C_{\varphi^{\prime}}$ implies improving the $O\left(m^{k}\right)$ algorithm for $M C_{\varphi}$.

(2) If $M C_{\varphi^{\prime}}$ belongs to one of $M C(\exists \exists \exists), M C(\forall \forall \forall), M C(\forall \exists \exists), M C(\exists \forall \forall)$, then we solve it directly in time $O\left(\mathrm{~m}^{3 / 2}\right)$, using ideas similar to triangle detection algorithms. If $\varphi^{\prime}$ has the quantifier structure $\forall \exists \forall$ (or its negated form $\exists \forall \exists$ ), then we reduce $M C_{\varphi^{\prime}}$ to $M C_{\varphi^{\prime \prime}}$, where $\varphi^{\prime \prime}$ has quantifier structure $\exists \exists \forall$, using Lemma 8.1. Otherwise, $\varphi^{\prime}$ is already in $\exists \exists \forall$ or equivalent form.

(3) We reduce a general model checking problem for $\varphi^{\prime \prime}$ of the quantifier structure $\exists \exists \forall$ to a graph property problem of the same quantifier structure.

(4) Using Lemma 5.6, we reduce formulas of form $\exists \exists \forall$ to a "hybrid" problem, which by Lemma 5.5 can be reduced to a combination of Sparse OV, Set Containment and 2-Set Cover (which we call Basic Problems). 
(5) We use a "universe-shrinking" technique (Lemmas 5.2, 6.1, and 6.2) on each of the Basic Problems, to transform a sparse instance into an equivalent one of small dimension.

(6) After applying this to the Hybrid Problem, we can complement edge relations as needed to transform all Basic Problems into OV (Lemmas 5.4 and 6.3).

(7) By applying the References $[3,16]$ algorithm to the instance of low-dimension OV, we get an improved algorithm.

Figure 2 shows a diagram of the above reduction process.

Moreover, Lemmas 8.1, 5.6, 5.5, and 5.1 also work for any constant $k \geq 2$. So for a problem in $M C\left(\exists^{k} \forall\right)$ or $M C\left(\forall \exists^{k-1} \forall\right)$, we can reduce it to $k$-OV as follows:

(1) If the problem belongs to $M C\left(\forall \exists^{k-1} \forall\right)$, then reduce it to $M C\left(\exists^{k} \forall\right)$ using Lemma 8.1.

(2) Eliminate hyperedges, then reduce the $M C\left(\exists^{k} \forall\right)$ to the Hybrid problem using Lemma 5.6.

(3) Reduce from the Hybrid Problem to a combination of $2^{k}$ Basic Problems, using Lemma 5.5.

(4) Reduce all Basic Problems to $k$-OV, using Lemma 5.1.

This completes the proof of Theorem 1.3.

\section{THE BUILDING BLOCKS}

Before the formal presentation of the reduction algorithms, this section gives an intuitive and highlevel view of the techniques used to reduce a first-order property problem to OV, in the proofs of Theorems 1.2, 1.3, and 1.4. Because of Lemma 1.1, in the remainder of this article, unless specified, we will use "OV" and " $k$-OV" to refer to sparse versions of these problems.

\subsection{Complementing Sparse Relations}

Recall the definitions of the $k$-Empty Intersection, $k$-Set Cover and Set Containment problems ${ }^{7}$ from Section 2.3. These problems have very similar structure: given set families $\mathcal{S}_{1} \ldots \mathcal{S}_{k}$ containing sets over elements of the universe $U$, each of them asks whether there is a tuple of sets, one in each family, such that a formula is satisfied for every element $u$ of the universe. Moreover, the formulas themselves are disjunctions of $u \in S_{i}$ or $u \notin S_{i}$, with one predicate for each $i$. The only difference is the polarity of the $\in$ relation (whether or not it is negated). We will refer to these types of problems as the Basic Problems; they will be our main building blocks.

For $k=2$, this gives us four basic problems: Set Disjointness, 2-Set Cover and two versions of Set Containment (direct and reversed). In each of them, the input consists of two set families $\mathcal{S}_{1}, \mathcal{S}_{2}$ of sizes $n_{1}, n_{2}$, respectively, and the universe $U$ of size $n_{u}$. The goal is to decide if there exist sets $S_{1} \in \mathcal{S}_{1}$ and $S_{2} \in \mathcal{S}_{2}$ such that for every $u \in U$, the corresponding formula $\psi_{\ell}$ holds. Here, $\ell \in\{00,01,10,11\}$ codes the sequence of polarities of occurrences of $\in$. This naturally generalizes to arbitrary $k$, with a Basic Problem for each $\ell \in\{0,1\}^{k}$; see Section 5.1 for formal definitions.

That is, Set Disjointness, 2-Set Cover and Set Containment can be stated as follows. Decide if $\exists S_{1} \in \mathcal{S}_{1} \exists S_{2} \in \mathcal{S}_{2} \forall u \in U \psi_{\ell}$ holds, where $\psi_{\ell}$ is as follows:

Set Disjointness: There is no common element in $S_{1}$ and $S_{2}: \psi_{\ell}=\psi_{00}=\neg\left(u \in S_{1}\right) \vee \neg\left(u \in S_{2}\right)$. 2-Set Cover: Union of $S_{1}$ and $S_{2}$ covers all of $U: \psi_{\ell}=\psi_{11}=\left(u \in S_{1}\right) \vee\left(u \in S_{2}\right)$.

\footnotetext{
${ }^{7} \mathrm{OV}$ is also 2-EI or Set Disjointess: $k$-EI is equivalent to $k$-OV, where vectors are represented by sets containing their $1 \mathrm{~s}$ Set Containment is equivalent to the Sperner Family, and $k$-Set Cover to $k$-Dominating Set under linear-time reductions.
} 
Set Containment: For $S_{1} \subseteq S_{2}, \psi_{\ell}=\psi_{01}=\neg\left(u \in S_{1}\right) \vee\left(u \in S_{2}\right)$. Similarly, in reversed Set Containment with $S_{2} \subseteq S_{1}, \psi_{\ell}=\psi_{10}=\left(u \in S_{1}\right) \vee \neg\left(u \in S_{2}\right)$.

All these problems are first-order properties: We can use unary relations to partition the vertex set into $\left(\mathcal{S}_{1}, \ldots, \mathcal{S}_{k}, U\right)$, and consider the relation " $\in$ " as a binary relation. We will use the context of hypergraphs to describe the input structure, as in Section 2.2. We let $n$ (corresponding to the number of vertices in the input graph) be the sum of $n_{1}, \ldots, n_{k}$ and $n_{u}$, and let the input size $m$ (corresponding to the number of edges in the input graph) be the sum of all sets' sizes in all set families. Borassi et al. [11] showed that when $k=2$, these Basic Problems require time $m^{2-o(1)}$ under SETH, and that if the size of universe $U$ is poly-logarithmic in the input size, then the three problems are equivalent under subquadratic-time reductions. The main idea of the reductions between these problems is to complement all sets in $\mathcal{S}_{1}$, or $\mathcal{S}_{2}$, or both. It is easy to see that $S_{1} \cap S_{2}=$ $\emptyset \Longleftrightarrow S_{1}{ }^{\complement} \cup S_{2}{ }^{\complement}=U \Longleftrightarrow S_{1} \subseteq S_{2}{ }^{\complement} \Longleftrightarrow S_{2} \subseteq S_{1}{ }^{\complement}$. Therefore, if we could complement the sets, we can easily prove the equivalences between the three Basic Problems. However, we cannot do this when $n_{u}$ is large.

For a sparse binary relation such as $\left(u \in S_{1}\right)$, we say that its complement, such as $\left(u \notin S_{1}\right)$, is cosparse. Suppose we want to enumerate all tuples $\left(S_{1}, u\right)$ s.t. $u \in S_{1}$; for that, we can go through all relations (aka edges) between $U$ and $\mathcal{S}_{1}$, which takes time linear in $m$. On the contrary, if we want to enumerate all pairs $\left(S_{1}, u\right)$ s.t. $u \notin S_{1}$, we cannot do this in linear time, because we cannot touch the pairs by touching edges between them. Moreover, when $n_{u}$ is as large as $\Omega(n)$, the number of such pairs can reach $\Theta\left(m^{2}\right)$. When $k=2$, a fine-grained reduction between $O\left(m^{2}\right)$-time problems allows neither quadratic time reductions, nor quadratic size problem instances.

Because of the above argument, it is hard to directly reduce between the Basic Problems, so instead we reduce each problem to a highly asymmetric instance of the same problem, where sparse relations are easily complemented to relations that are also sparse. Observe that when the size of universe $U$ is small enough, complementing all sets can be done in time $O(m \cdot|U|)$, which can be substantially faster than $O\left(m^{2}\right)$. The new instance created also has size $O(m \cdot|U|)$, so that it is only slightly larger than $m$. So by carefully choosing the size of $U$, we can construct truly subquadratic time reduction algorithms that preserve the improved factor in running time. Using this technique that we call universe-shrinking self-reduction, we can show that OV, 2-Set Cover and Set Containment are equivalent under fine-grained reductions.

The self-reduction employs the "high-degree low-degree" trick, which has been also used in other sparse graph algorithms [7]. First, consider sets of large cardinality: there cannot be too many of them, because the structure is sparse. Thus, we can do exhaustive search over these sets to check if any of them is in a solution. For sets of small cardinality, we hash the universe $U$ to a smaller universe, where complementing the sets does not take too much time and space. From this reduction, the claim follows:

Claim 4.1. If any one of OV, 2-Set Cover and Set Containment has truly subquadratic time algorithms, then the other two are also solvable in subquadratic time. Thus these problems are all hard for $M C(3)$.

Claim 4.1 is itself an interesting result: In Reference [11], conditional lower bounds for many problems stem from the above three problems, forming a tree of reductions. By our equivalence, the root of the tree can be replaced by the quadratic-time hardness conjecture on any of the three problems, simplifying the reduction tree. Claim 4.1 also shows that an improved algorithm for any of these three problems implies improved algorithms for the other two.

Claim 4.1 is proven by derandomizing Lemma 5.1 for $k=2$; see Section 6 for details. In Section 5 , we give randomized reductions for an arbitrary $k$. 


\subsection{Sparse and Co-sparse Relations}

Having shown how to reduce any two Basic Problems with the same $k$ to each other, we will now reduce generic first-order properties to the Basic Problems. The detailed processes are complicated, so here we start with a high-level idea in reductions and algorithm design throughout the article.

Our algorithms often need to iterate over all tuples or pairs $\left(x_{i}, x_{j}\right)$ satisfying some conditions, to list such tuples, or to count the number of them, performing first-order query processing. A set of such tuples (pairs) $\left(x_{i}, x_{j}\right)$ can be considered a result of a first-order query defined by an intermediate formula $\varphi^{\prime}$ on the (hyper)graph $G$ (or some intermediate structures). Our reduction algorithms often generate such queries, evaluate them, and combine the results (e.g., by counting) to compute the solutions.

There are three possible outcomes of such queries: the result can be a sparse set of tuples, a cosparse set, or neither. If the result of the query is a sparse relation such as $\left[R_{1}\left(x_{1}, x_{2}\right) \wedge \neg R_{2}\left(x_{1}, x_{2}\right)\right]$, then we can iterate over the tuples (say, first enumerate all pairs satisfying $R_{1}\left(x_{1}, x_{2}\right)$, then check for which of them $R_{2}\left(x_{1}, x_{2}\right)$ is false). Then, we can do further operations on the sparse set of $\left(x_{1}, x_{2}\right)$ tuples resulting from the query. When the result of the query is a co-sparse set such as for $\left[\neg R_{1}\left(x_{1}, x_{2}\right) \wedge \neg R_{2}\left(x_{1}, x_{2}\right)\right]$, we cannot directly iterate over pairs satisfying the query. Instead, we work on its complement (which is sparse, instead of co-sparse), but then do some further processing to filter out those pairs from future use (say, find all pairs $\left(x_{1}, x_{2}\right)$ so that at least one of $R_{1}\left(x_{1}, x_{2}\right)$ or $R_{2}\left(x_{1}, x_{2}\right)$ is true, then exclude those pairs from future use). Sometimes, the result of a query is neither sparse nor co-sparse, but we will show it is always a combination of sparse and co-sparse relations. Thus, we need to distinguish them and deal with the sparse and co-sparse parts separately.

We exemplify this process by considering the query $\left[\neg R_{1}\left(x_{1}, x_{2}\right) \vee \neg R_{2}\left(x_{1}, x_{2}\right)\right]$. For a pair $\left(x_{1}, x_{2}\right)$, to make the formula true, predicates $R_{1}, R_{2}$ can be assigned values from $\{($ True, False $)$, (False, True), (False, False $)\}$. In the first two cases, the sets of pairs $\left(x_{1}, x_{2}\right)$ satisfying $\left[R_{1}\left(x_{1}, x_{2}\right) \wedge\right.$ $\left.\neg R_{2}\left(x_{1}, x_{2}\right)\right]$ and $\left[\neg R_{1}\left(x_{1}, x_{2}\right) \wedge R_{2}\left(x_{1}, x_{2}\right)\right]$ are sparse, while in the last case, the set of pairs satisfying $\left[\neg R_{1}\left(x_{1}, x_{2}\right) \wedge \neg R_{2}\left(x_{1}, x_{2}\right)\right]$ is co-sparse. So if we want to work on the tuples satisfying this query, we list tuples satisfying the first two cases directly by enumerating edges, and enumerate the tuples not satisfying the third case (i.e., the tuples, where either $R_{1}\left(x_{1}, x_{2}\right)$ or $R_{2}\left(x_{1}, x_{2}\right)$ is true), to exclude them from future use.

In general, a query can be written as a DNF, where the result of each term is a conjunction of predicates and negated predicates, and therefore either sparse or co-sparse. Then we can deal with the sparse and co-sparse cases separately. We will use this technique for constructing the Hybrid Problem in Section 5.2.

Now, we would like to reduce $M C_{\varphi}$ to OV for an arbitrary $\varphi=(\exists x)(\exists y)(\forall z) \psi(x, y, z)$. First, suppose that all predicates $R_{1} \ldots R_{r}$ in $\psi$ are at most binary, and all binary predicates involve $z$. One attempt is to create a set $S_{x}$ for each element $x$ and a set $S_{y}$ for each element $y$. Then, we create elements in universe $U$ by creating $2^{r}$ elements $u_{\left(z, 0^{r}\right)}, \ldots, u_{\left(z, 1^{r}\right)}$ for each $z$, where $r$ is the number of different predicates in $\psi$, and the length- $r$ strings in the subscripts correspond to the $2^{r}$ truth assignments of all these predicates. We construct the sets so that $S_{x}$ (or $S_{y}$ ) contains element $u_{(z, a)}$ iff the assignment $a$ falsifies $\psi$ and the relations between $x$ (or $y$ ) and $z$ agree with $a$. In this way, sets $S_{x}$ and $S_{y}$ both contain some element $u_{(z, a)}$ in $U$ iff there is some $z$ such that $x, y, z$ do not satisfy $\psi$. Then, if there exists such pair of disjoint sets $S_{x}$ and $S_{y}$, the corresponding $x$ and $y$ satisfy that for all $z, \psi$ is true.

However, we cannot touch all $z$ 's for each $x$ or $y$ for creating this instance in substantially less than $n^{2}$ time. So, we divide the relations of this Set Disjointness instance into sparse and co-sparse 
ones. For that, we introduce a Hybrid Problem that is a combination of Basic Problems. Depending on the four combinations of sparsity or co-sparsity on the relations between variables $x, z$ and $y, z$, we reduce $M C_{\varphi}$ not only to OV, but to a combination of OV, Set Containment, reversed Set Containment (i.e., finding $S_{2} \subseteq S_{1}$ instead of $S_{1} \subseteq S_{2}$ ), and 2-Set Cover. (Namely, the sub-problem Set Disjointness deals with the case, where the relations between $x$ and $z$ and between $y$ and $z$ are both sparse; the sub-problems Set Containment, reversed Set Containment and 2-Set Cover deals with the cases, where these relations are sparse and co-sparse, co-sparse and sparse, co-sparse and co-sparse, respectively.) We decide if there is a pair of sets being the solutions of all sub-problems. Finally, because these Basic Problems can be reduced to each other, we can use the algorithm for $\mathrm{OV}$ to solve the instance of the Hybrid Problem, and then to solve $M C_{\varphi}$.

This approach takes care of binary predicates involving $z$; to handle relations among existentially quantified variables, additional tools are needed. Thus, the Hybrid Problem definition also involves a relation $R(x, y)$ and a "sparsity type" designation, specifying whether $R$ codes a sparse relation between $x$ and $y$, or its sparse complement. However, this additional information can be modeled by adding new elements to the universe and strategically placing them in the corresponding sets, thus reducing the more complex case to a combination of four Basic Problems.

See Lemma 5.6 for the proof that covers more complicated cases.

\section{COMPLETENESS OF $K$-OV IN $M C\left(\exists^{K} \forall\right)$}

This section will prove the completeness of $k$-OV in $M C\left(\exists^{k} \forall\right)$ problems. Here we only consider the input structures that are graphs, i.e., where all relations are either unary or binary; see Section 7 of this article for the reduction from hypergraphs to graphs. First, we introduce a class of Basic Problems, and prove that these problems are equivalent to $k$-OV under exact complexity reductions. Then, we show that any problem in $M C\left(\exists^{k} \forall\right)$ can be reduced to a combination of Basic Problems (a.k.a. the Hybrid Problem).

\subsection{How to Complement a Sparse Relation: Basic Problems and Reductions between Them}

In this section, we define the Basic Problems for $k \geq 2$, generalizing $k$-OV, $k$-Set Cover and Set Containment problems, and prove that these problems are fine-grained reducible to each other under randomized reductions. In Section 6, we will give deterministic reductions for $k=2$.

Let $k \geq 2$. We introduce $2^{k}$ Basic Problems labeled by $k$-bit binary strings from $0^{k}$ to $1^{k}$. The input of these problems is the same as that of $k$-EI defined in Section 2.3: $k$ set families $\mathcal{S}_{1} \ldots \mathcal{S}_{k}$ of size $n_{1}, \ldots, n_{k}$ on a universe $U$ of size $n_{u}$. We define $2^{k}$ quantifier-free formulas $\psi_{0^{k}}, \ldots, \psi_{1^{k}}$ such that

$$
\psi_{\ell}=\left(\bigvee_{i, \ell[i]=0}\left(\neg\left(u \in S_{i}\right)\right)\right) \vee\left(\bigvee_{i, \ell[i]=1}\left(u \in S_{i}\right)\right)
$$

Here, $i \in\{1, \ldots, k\}$ and $\ell[i]$, the $i$ th bit of label $\ell$, specifies whether $u$ is in each $S_{i}$ or not in the $i$ th term of $\psi \ell$.

For each $\ell$, let $\varphi_{\ell}=\left(\exists S_{1} \in \mathcal{S}_{1}\right) \ldots\left(\exists S_{k} \in \mathcal{S}_{k}\right)(\forall u \in U) \psi_{\ell}$. For simplicity, we will omit the domains of the variables in these formulas. We call $M C_{\varphi_{0} k}, \ldots, M C_{\varphi_{1 k}}$ the Basic Problems. We refer to the Basic Problem $M C_{\varphi_{\ell}}$ as $B P[\ell]$. These problems are special cases of first-order model checking on graphs, where sets and elements correspond to vertices, and membership relations correspond to edges. Note that $B P\left[0^{k}\right]$ is $k$-EI, and $B P\left[1^{k}\right]$ is $k$-Set Cover. When $k=2, B P[01]$ and $B P[10]$ are Set Containment problems, and $B P[00]$ is the Set Disjointness problem. For a $k$-tuple 
$\left(S_{1} \in \mathcal{S}_{1}, \ldots, S_{k} \in \mathcal{S}_{k}\right)$ satisfying $(\forall u) \psi_{\ell}$, we call it a solution of the corresponding Basic Problem $B P[\ell]$.

We present a randomized ${ }^{8}$ fine-grained mapping reduction between any two Basic Problems, thus proving the following lemma, which generalizes Claim 4.1 to $k>2$.

Lemma 5.1. Let $s(m)$ be a non-decreasing function such that $2^{\Omega(\sqrt{\log m})} \leq s(m)<m^{1 / 5}$. For any $\ell_{1}, \ell_{2} \in\{0,1\}^{k}$, there is a randomized exact complexity reduction $\left(B P\left[\ell_{1}\right], m^{k} /(s(m))^{1 / 6}\right) \leq_{E C}$ $\left(B P\left[\ell_{2}\right], m^{k} / s(m)\right)$.

For problems $B P\left[\ell_{1}\right]$ and $B P\left[\ell_{2}\right]$, where $\ell_{1}$ and $\ell_{2}$ only differ in the $i$ th bit, if we are allowed to complement all sets in $\mathcal{S}_{i}$, we can easily reduce between them. Similarly, if $\ell_{1}$ and $\ell_{2}$ differ in more than one bit, we can complement all the sets in corresponding set families. However, complementing the sets in $\mathcal{S}_{i}$ takes time $O\left(n_{i} n_{u}\right)$, which might be as large as $\Theta\left(m^{2}\right)$. To solve this, we self-reduce $B P\left[\ell_{1}\right]$ on the universe $U$ to the same problem on a smaller universe $U^{\prime}$, and then complement sets on $U^{\prime}$. For any given $\delta$, if the size of $U^{\prime}$ is $n_{u}^{\prime}=O\left(m^{\delta}\right)$, then complementing all sets in $\mathcal{S}_{i}$ only takes time and space $m \cdot O\left(m^{\delta}\right)=O\left(m^{1+\delta}\right)$.

Lemma 5.2. (Randomized universe-shrinking self-reductions of Basic Problems)

Let label $\ell$ be any binary string in $\{0,1\}^{k}$. For any $s(m)=2^{\Omega(\sqrt{\log m})}$, given a $B P[\ell]$ instance $I$ of size $m$ and universe $U$ of size $n_{u}$, we can either solve it in time $O\left(m^{k} / s(m)\right)$, or use time $O\left(m^{k} / s(m)\right)$ to create a $B P[\ell]$ instance $I^{\prime}$ of size $O\left(m \cdot s(m)^{5}\right)$ on universe $U^{\prime}$ whose size is $n_{u}^{\prime}=O\left(s(m)^{5}\right)$, so that $I \in B P[\ell]$ iff $I^{\prime} \in B P[\ell]$ with error probability bounded by $O(1 / s(m))$.

Note that the self-reduction of $k$-OV actually reduces the Sparse $k$-OV to a moderate-dimension version of $k$-OV, implying Lemma 1.1. The other direction (moderate-dimension $k$-OV to Sparse $k$-OV) is easy since if the dimension $d=n^{\delta}$, then $m$ is at most $d \cdot n=n^{1+\delta}$, as required.

COROLlARY 5.3. (Reverse direction of Lemma 1.1)

Suppose that for any $k \geq 2$ there exists $\delta, \epsilon>0$ and a (randomized) $O\left(n^{k-\epsilon}\right)$ algorithm solving $k-O V$ with dimension $d=n^{\delta}$. Then there is an $\epsilon^{\prime}>0$ and a (randomized) $O\left(m^{k-\epsilon^{\prime}}\right)$ time algorithm solving Sparse $k-O V$.

Proof. The algorithm converts an instance of Sparse $k$-OV to an instance of $k$-OV of dimension $n^{\delta}$ using universe-shrinking self-reduction (Lemma 5.2) and then applies assumed $O\left(n^{k-\epsilon}\right)$ time algorithm to the reduced instance. More specifically, let $m=O\left(n^{1+\gamma}\right)$, where $n$ is the number of vectors. Choosing $s(m)=O\left(m^{\delta / 5(1+\gamma)}\right)$ for some $\delta>0$ creates an instance of OV with dimension $n_{u}^{\prime}=O\left(s(m)^{5}\right)=O\left(n^{\delta}\right)$, and size $m^{\prime}=O\left(n^{1+\delta+\gamma}\right)$; number of vectors $n$ remains unchanged. Now, the reduction takes time $O\left(m^{k} /(s(m))^{5}\right)=O\left(m^{k-\delta /(1+\gamma)}\right)$, and running the $O\left(n^{k-\epsilon}\right)$ time algorithm on the reduced instance takes $O\left(n^{k-\epsilon}\right) \leq O\left(m^{k-\epsilon /(1+\gamma)}\right)$ time. Setting $\epsilon^{\prime}=\min \{\delta /(1+\gamma), \epsilon /(1+$ $\gamma)\}$ completes the proof.

We will present the randomized self-reductions for problems $B P[\ell]$ s.t. $\ell \neq 1^{k}$ in Section 5.1.1. For $B P\left[1^{k}\right]$, we will prove that it is either easy to solve or easy to complement in Section 5.1.2.

After shrinking the universe, we complement the sets to reduce between two Basic Problems $B P\left[\ell_{1}\right]$ and $B P\left[\ell_{2}\right]$ according to the following lemma.

Lemma 5.4 (Reduction between different Basic Problems). For two different labels $\ell_{1}, \ell_{2} \in$ $\{0,1\}^{k}$, given set families $\mathcal{S}_{1}, \ldots, \mathcal{S}_{k}$, let $\mathcal{S}_{1}^{\prime}, \ldots, \mathcal{S}_{k}^{\prime}$ be defined such that

\footnotetext{
${ }^{8}$ The deterministic reduction will be presented in Section 6 .
} 


$$
\mathcal{S}_{i}^{\prime}= \begin{cases}\left\{S_{i}{ }^{\complement} \mid S_{i} \in \mathcal{S}_{i}\right\}, & \text { if } \ell_{1}[i] \neq \ell_{2}[i] \\ \mathcal{S}_{i}, & \text { otherwise }\end{cases}
$$

then, $\left(\exists S_{1} \in \mathcal{S}_{1}\right) \ldots\left(\exists S_{k} \in \mathcal{S}_{k}\right)(\forall u) \psi_{\ell_{1}}$ iff $\left(\exists S_{1}^{\prime} \in \mathcal{S}_{1}^{\prime}\right) \ldots\left(\exists S_{k}^{\prime} \in \mathcal{S}_{k}^{\prime}\right)(\forall u) \psi_{\ell_{2}}$.

The proof of correctness is straightforward.

Proof of Lemma 5.1. Pick $s^{\prime}(m)=s(m)^{1 /(6 k)}$. Using Lemma 5.2, we shrink the universe to size $n_{u}^{\prime}=s^{\prime}(m)^{5}$. So the time complexity in this step is bounded by $O\left(m \cdot s^{\prime}(m)^{5}\right)$, which is significantly less than $m^{k} / s(m)$ even if $k=2$.

Let new instance size be $m^{\prime}$. So $m^{\prime}=m \cdot s^{\prime}(m)^{5}$. Given that the constructed instance can be decided in time $m^{\prime k} / s\left(m^{\prime}\right)$, we get $m^{\prime k} / s\left(m^{\prime}\right)<\left(m\left(s(m)^{1 /(6 k)}\right)^{5}\right)^{k} / s(m)<m^{k} / s(m)^{1 / 6}$. Thus, by the two-step fine-grained mapping reductions given by Lemma 5.2 and Lemma 5.4, we have an exact complexity reduction between any two Basic Problems, completing the proof for Lemma 5.1.

5.1.1 Randomized Universe-shrinking Self-reduction of $B P[\ell]$ Where $\ell \neq 1^{k}$. This section proves part of Lemma 5.2, by giving a randomized universe-shrinking self-reduction of $B P[\ell]$, where $\ell \neq$ $1^{k}$. The main idea is to divide the sets into large and small ones. For large sets, there are not too many of them in the sparse structure, so we can work on them directly. For small sets, we use a Bloom Filter mapping each element in $U$ to some elements in $U^{\prime}$ at random, and then for each set on universe $U$, we compute the corresponding set on universe $U^{\prime}$. Next we can decide the same problem on these newly computed sets, instead of sets on $U$. (Reference [17] used a similar technique in reducing from Orthogonal Range Search to the Subset Query problem.) Because the sets are small, it is unlikely that some elements in two different sets on $U$ are mapped to the same element on $U^{\prime}$, bounding the error probability.

- Step 1: Large sets. Let $d=s(m)$. For sets of size at least $d$, we directly check if they are in any solutions. There are at most $O(\mathrm{~m} / \mathrm{d})=O(\mathrm{~m} / \mathrm{s}(\mathrm{m}))$ of such large sets. In the outer loop, we enumerate all large sets in $\mathcal{S}_{1}, \ldots, \mathcal{S}_{k}$. If their sizes are pre-computed, then we can do the enumeration in $O(\mathrm{~m} / \mathrm{s}(\mathrm{m}))$. Assume the current large set is $S_{i} \in \mathcal{S}_{i}$. Because variables quantified by $\exists$ are interchangeable, we can interchange the order of variables, and let $S_{i}$ be the outermost quantified variable $S_{1}$. On each such $S_{i}$ (or $S_{1}$ after interchanging), we create a new formula $\psi_{S_{1}}$ on variables $S_{2}, \ldots, S_{k}, u$ from formula $\psi$, by replacing $u \in S_{1}\left(u \notin S_{1}\right)$ by a unary relation on $u$. Then, we decide if the graph induced by $\mathcal{S}_{2}, \ldots, \mathcal{S}_{k}$ and $U$ satisfies $\left(\exists S_{2}\right) \ldots\left(\exists S_{k}\right)(\forall u) \psi_{S_{1}}$, using the baseline algorithm, which takes time $O\left(m^{k-1}\right)$ for each such large set $S_{1}$. Thus the overall running time is $O(\mathrm{~m} / \mathrm{s}(\mathrm{m})) \cdot O\left(\mathrm{~m}^{k-1}\right)=O\left(\mathrm{~m}^{k} / \mathrm{s}(\mathrm{m})\right)$. If no solution is found in this step, then proceed to Step 2.

- Step 2: Small sets. Now we can exclude all the sets of size at least $d$. For sets of size smaller than $d$, we do the self-reduction to universe $U^{\prime}$ of size $n_{u}^{\prime}=s(m)^{5}$. Let $t=s(m)$, and let $h: U \rightarrow U^{\prime t}$ be a function that independently maps each element $u \in U$ to $t$ elements in $U^{\prime}$ at random. On set $S \subseteq U$, we overload the notation $h$ by defining $h(S)=\bigcup_{u \in S} h(u)$. For all set families $\mathcal{S}_{i}$, we compute new sets $h\left(S_{i}\right)$ for all $S_{i} \in \mathcal{S}_{i}$. Then, we decide whether the new sets satisfy the following sentence, which is another $B P[\ell]$ problem:

$$
\left(\exists S_{1}\right) \ldots\left(\exists S_{k}\right)(\forall u) \bigvee_{i, \ell[i]=0} \neg\left(u \in h\left(S_{i}\right)\right) \vee \bigvee_{i, \ell[i]=1}\left(u \in h\left(S_{i}\right)\right)
$$

The size of the new instance is $O(n t)=O(m \cdot s(m))$, and the running time of the selfreduction is also $O(n t)=O(m \cdot s(m))$. So it is a fine-grained mapping reduction for any $k \geq 2$. 


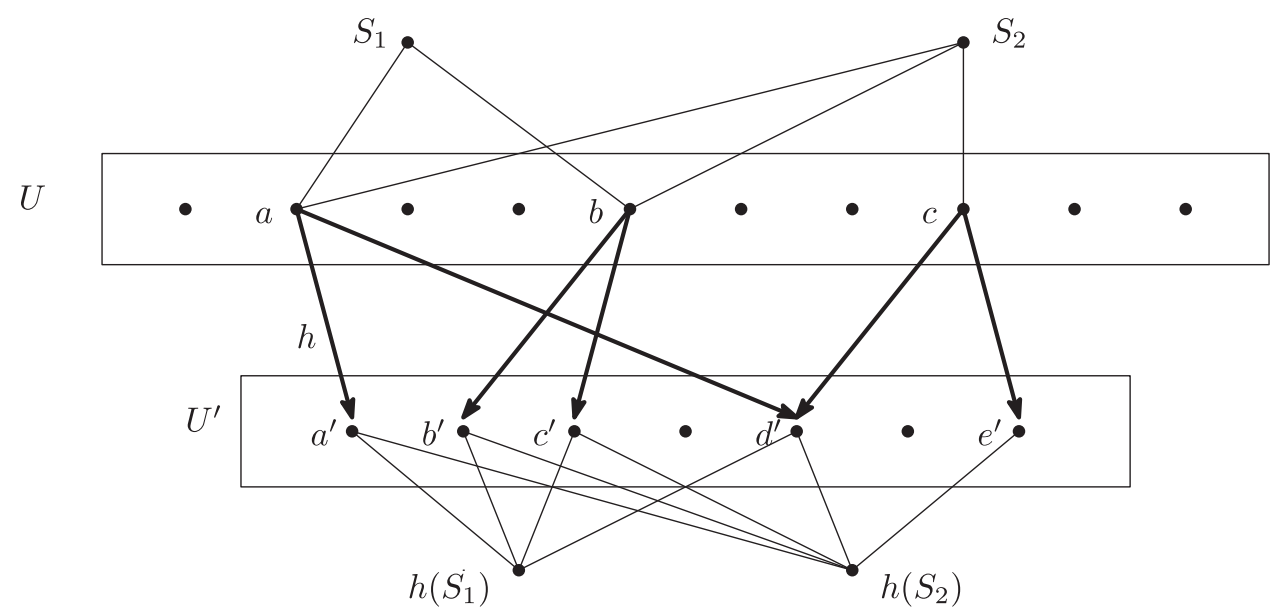

Fig. 3. The universe-shrinking process. $S_{1}=\{a, b\}$ and $S_{2}=\{a, b, c\}$. After the mapping $h$, the new sets are $h\left(S_{1}\right)=\left\{a^{\prime}, b^{\prime}, c^{\prime}, d^{\prime}\right\}$ and $h\left(S_{2}\right)=\left\{a^{\prime}, b^{\prime}, c^{\prime}, d^{\prime}, e^{\prime}\right\}$.

Figure 3 illustrates an example of the universe-shrinking self-reduction for $B P[01]$, where we look for $S_{1}, S_{2}$ so that $S_{1} \subseteq S_{2}$. If they exist, then after the self-reduction, it is always true that $h\left(S_{1}\right) \subseteq h\left(S_{2}\right)$. Still, it might happen that some $S_{1} \nsubseteq S_{2}$ but $h\left(S_{1}\right) \subseteq h\left(S_{2}\right)$. In this case, a false positive occurs. In $B P[00]$, a false negative may occur when there are two disjoint sets, but some elements in $S_{1} \cap S_{2}$ are mapped to the same element in $U^{\prime}$. Next we will analyze the error probability of this reduction.

Analysis. Because variables quantified by $\exists$ are interchangeable, w.l.o.g. for $\ell$ containing $i(i \geq 1)$ zeros and $k-i$ ones, assume $B P[\ell]$ is defined by

$$
\left(\exists S_{1}\right) \ldots\left(\exists S_{k}\right)(\forall u)\left[\left(\bigvee_{j=1}^{i}\left(u \notin S_{j}\right)\right) \vee\left(\bigvee_{j=i+1}^{k}\left(u \in S_{j}\right)\right)\right]
$$

equivalently, $\left(\exists S_{1}\right) \ldots\left(\exists S_{k}\right)\left[\left(\bigcap_{j=1}^{i} S_{j}\right) \subseteq\left(\bigcup_{j=i+1}^{k} S_{j}\right)\right]$.

Let sets $A=\bigcap_{j=1}^{i} S_{j}$ and $B=\bigcup_{j=i+1}^{k} S_{j}$. Then the problem is to decide whether there exists $\left(S_{1}, \ldots, S_{k}\right)$ so that $A \subseteq B$. After the self-reduction, let $A^{\prime}=\bigcap_{j=1}^{i} h\left(S_{j}\right)$ and $B^{\prime}=\bigcup_{j=i+1}^{k} h\left(S_{j}\right)$, and decide if there exists $\left(S_{1}, \ldots, S_{k}\right)$ such that $A^{\prime} \subseteq B^{\prime}$.

- False positive. A false positive occurs when $\forall\left(S_{1}, \ldots, S_{k}\right), A \nsubseteq B$, but $\exists\left(S_{1}, \ldots, S_{k}\right), A^{\prime} \subseteq$ $B^{\prime}$. For a fixed tuple $\left(S_{1}, \ldots, S_{k}\right)$ such that $A \nsubseteq B$, an error occurs when $h(u) \subseteq B^{\prime}$ for all $u \in A-B$. The size of $B^{\prime}$ is at most $k d t$. So the error probability $\operatorname{Pr}\left[h(u) \subseteq B^{\prime}\right] \leq\left(k d t / n_{u}^{\prime}\right)^{t}=$ $\left(k s(m) \cdot s(m) / s(m)^{5}\right)^{t}<s(m)^{-t}$. The size of $A-B$ is bounded by $k d$, so the probability $\operatorname{Pr}\left[\exists u \in A-B, h(u) \subseteq B^{\prime}\right] \leq k d \cdot s(m)^{-t}$. There are $O\left(m^{k}\right)$ tuples of $\left(S_{1}, \ldots, S_{k}\right)$, so the total error probability is at most $O\left(m^{k}\right) \cdot k d \cdot s(m)^{-t}=O\left(m^{k} \cdot s(m) / s(m)^{s(m)}\right)$, which is exponentially small.

- False negative. A false negative occurs when $\exists\left(S_{1}, \ldots, S_{k}\right), A \subseteq B$, but $\forall\left(S_{1}, \ldots, S_{k}\right), A^{\prime} \nsubseteq$ $B^{\prime}$. Fix any tuple $\left(S_{1}, \ldots, S_{k}\right)$ that satisfies $A \subseteq B$ in the original instance, and consider the distribution on the corresponding $h\left(S_{1}\right), . ., h\left(S_{k}\right)$. By definition, $B^{\prime}=\bigcup_{u \in B} h(u)$, and so contains $\bigcup_{u \in A} h(u)$. So if $A^{\prime} \subseteq \bigcup_{u \in A} h(u)$, we will have $A^{\prime} \subseteq B^{\prime}$, and there will not be a false negative. If not, then there is some $u^{\prime} \in A^{\prime}=\bigcap_{j=1}^{i} h\left(S_{j}\right)$, such that $u^{\prime} \notin \bigcup_{u \in A} h(u)$. Then for each $j \in\{1, \ldots, i\}$, in each $S_{j}$ there is a $u_{j} \in S_{j}$ with $u^{\prime} \in h\left(u_{j}\right)$, but not all $u_{j}$ are identical. 
(Otherwise the $u_{j} \in A$, so $u^{\prime} \in h\left(u_{j}\right) \subseteq \bigcup_{u \in A} h(u)$, contradicting $u^{\prime} \notin \bigcup_{u \in A} h(u)$ ). In particular, this means that for some $j_{1}, j_{2}$, there are $u_{j_{1}} \in S_{j_{1}}, u_{j_{2}} \in S_{j_{2}}$, such that $h\left(u_{j_{1}}\right) \cap h\left(u_{j_{2}}\right) \neq$ $\emptyset$. So the error probability is bounded by $k^{2} \cdot \operatorname{Pr}\left[\exists\left(u_{1} \in S_{j_{1}}, u_{2} \in S_{j_{2}}\right), h\left(u_{1}\right) \cap h\left(u_{2}\right) \neq \emptyset\right]$. Because $\left|S_{j_{1}}\right|$ and $\left|S_{j_{2}}\right|$ are at most $d$, by Birthday Paradox, the probability is at most $O\left(k^{2} d^{2} t^{2} / n_{u}^{\prime}\right)=O\left(s(m)^{-1}\right)$. This is the upper bound of the error probability for the fixed $\left(S_{1}, \ldots, S_{k}\right)$ tuple. Then, the probability of the event " $\forall\left(S_{1}, \ldots, S_{k}\right), A^{\prime} \nsubseteq B^{\prime \prime}$ " is even smaller.

5.1.2 Deterministic Universe-shrinking Self-reduction of $B P\left[1^{k}\right]$. This section proves the remaining part of Lemma 5.2 , by showing $B P\left[1^{k}\right]$ is either easy to solve or easy to complement. $B P\left[1^{k}\right]$ is the $k$-Set Cover problem, which decides whether there exist $k$ sets covering the universe $U$. It is special in the Basic Problems: When $n_{u}$ is small, the sets are easy to complement; when $n_{u}$ is large, the problem is easy to solve.

- Case 1: Large universe. If $n_{u}>s(m)$, then in a solution of this problem, at least one set has size at least $n_{u} / k$. There are at most $m /\left(k / n_{u}\right)=O(m / s(m))$ such large sets, thus they can be listed in time $O(m / s(m))$, after pre-computation on the sizes of all sets. Our algorithm exhaustively searches all such large sets. And then, similarly to "Step 1" in Section 5.1.1, for each of the large sets, we run the baseline algorithm to find the remaining $k-1$ sets in the $k$ set cover, which takes time $O\left(\mathrm{~m}^{k-1}\right)$. So the overall running time is $O(\mathrm{~m} / \mathrm{s}(\mathrm{m})) \cdot O\left(\mathrm{~m}^{k-1}\right)=$ $O\left(m^{k} / s(m)\right)$.

- Case 2: Small universe. If $n_{u} \leq s(m)$, then we do not need a universe-shrinking selfreduction, because the universe is already small enough.

\subsection{Hybrid Problem}

Next we reduce general $M C\left(\exists^{k} \forall\right)$ problems to an intermediate problem called the Hybrid Problem, which is a combination of $2^{k}$ Basic Problems. Then by reducing from the Hybrid Problem to Basic Problems, we can set up a connection between $M C\left(\exists^{k} \forall\right)$ and OV.

Let $k \geq 2$. The input to the Hybrid Problem is:

(1) Set families $\mathcal{S}_{1} \ldots \mathcal{S}_{k}$ defined on universe $U$, where $U$ is partitioned into $2^{k}$ disjoint subuniverses: $U=\bigcup_{\ell \in\{0,1\}^{k}} U_{\ell}$.

(2) A binary relation $R$ defined on pairs of sets from any two distinct set families. $R$ is a symmetric relation $\left(R\left(S_{i}, S_{j}\right)\right.$ iff $\left.R\left(S_{j}, S_{i}\right)\right)$.

(3) type is binary string of length $\left(\begin{array}{c}k \\ 2\end{array}\right)$, indexed by two integers $[i, j]$, s.t. $i, j \in\{1, \ldots, k\}$ and $i<j$.

The goal of the problem is to decide if there exist $S_{1} \in \mathcal{S}_{1}, \ldots, S_{k} \in \mathcal{S}_{k}$ such that both of the following constraints are true:

(A) For each $\ell \in\{0,1\}^{k},\left(S_{1}, \ldots S_{k}\right)$ is a solution of $B P[\ell]$ defined on sub-universe $U_{\ell}$.

(B) For all pairs of indices $i, j \in\{1, \ldots, k\}, i<j$, we have that $R\left(S_{i}, S_{j}\right)=$ true iff type $[i, j]=1$.

We let $n$ be the sum of $\left|\mathcal{S}_{1}\right|, \ldots,\left|\mathcal{S}_{k}\right|$ and $U$, and let $m$ be the number of tuples in all unary and binary relations. The Hybrid Problem is a first-order property on graphs with additional constraints. As usual, we assume all relations in the Hybrid Problem are sparse $\left(m \leq n^{1+o(1)}\right)$. Figure 4 shows a solution to a Hybrid Problem instance when $k=2$.

Intuition Behind the Hybrid Problem. In the Hybrid Problem, the set families $S_{1}, \ldots, S_{k}$ encode the conditions on relations involving $x_{k+1}$, while the binary relation $R$ and the types encode the conditions on relations not involving $x_{k+1}$. We mentioned in Section 4 that any first-order query containing two variables can be written in a "normal form," which is a combination of sparse and 


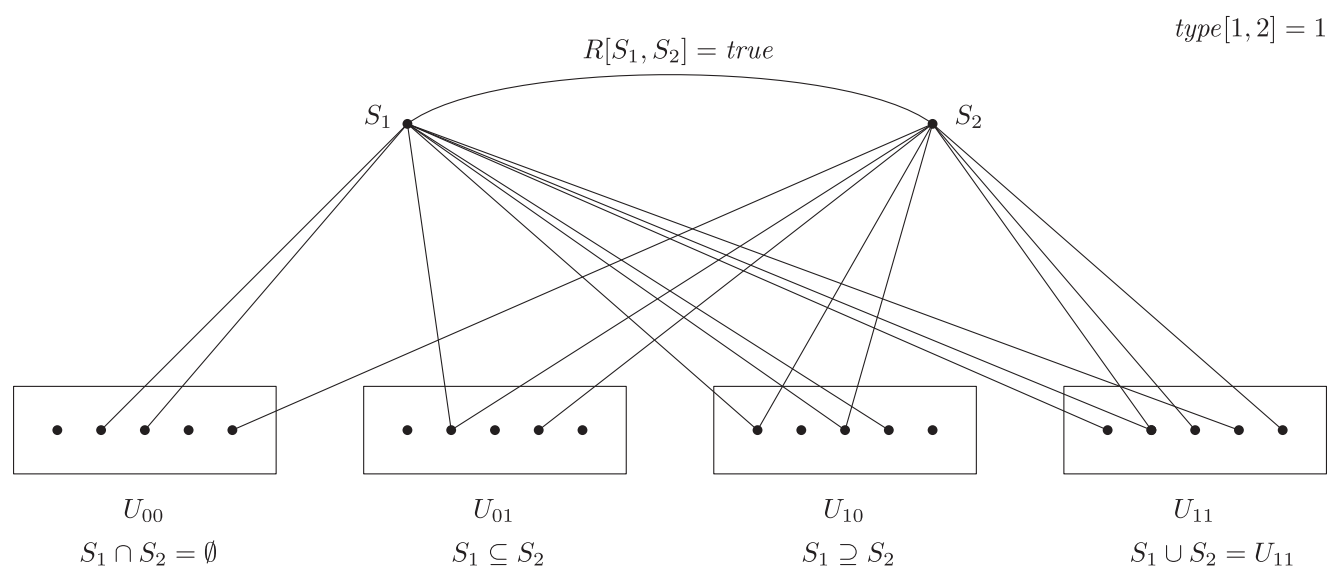

Fig. 4. An example of a solution to a Hybrid Problem instance, when $k=2$. In sub-universes $U_{00}, U_{01}, U_{10}, U_{11}$, sets $S_{1}$ and $S_{2}$ are solutions of $B P[00]$ (Set Disjointness), $B P[01]$ (Set Containment), $B P[10]$ (Set Containment in the reversed direction) and $B P[11](2$-Set Cover), respectively. Furthermore, type $[1,2]=1$ specifies that the predicate $R$ on $\left(S_{1}, S_{2}\right)$ must be true.

co-sparse relations. The Hybrid Problem is designed for separating sparse relations from co-sparse ones, for all pairs of variables in formula $\varphi$.

The relation between the pair of variables $\left(x_{i}, x_{k+1}\right)$, where $1 \leq i \leq k$ can be either sparse or co-sparse. Because there are $k$ such variables $x_{i}$, there are $2^{k}$ cases for a combination $\left(\left(x_{1}, x_{k+1}\right), \ldots,\left(x_{k}, x_{k+1}\right)\right)$. These cases correspond to the $2^{k}$ Basic Problems. In each Basic Problem, we deal with one of the $2^{k}$ cases.

For a relation between the pair of variables $\left(x_{i}, x_{j}\right)$, where $1 \leq i<j \leq k$, it also can be either sparse or co-sparse. We use type $[i, j]$ to distinguish the two cases: When it is set to 1 , we expect a sparse relation for $\left(x_{i}, x_{j}\right)$, otherwise a co-sparse relation.

\subsubsection{Reduction to Basic Problems.}

Lemma 5.5. Let $s(m)$ be a non-decreasing function such that $2^{\Omega(\sqrt{\log m})} \leq s(m)<m^{1 / 5}$. Then, (Hybrid Problem, $\left.m^{k} /(s(m))^{1 / 6}\right) \leq_{E C}\left(k-O V, m^{k} /(s(m))\right)$.

Given an instance of the Hybrid Problem, we can do the following modification in time $O(m)$. For each pair of indices $i, j$, where $1 \leq i<j \leq k$, we construct auxiliary elements depending on the value of type $[i, j]$.

- Case 1: If type $[i, j]=0$, then if a pair $S_{i} \in \mathcal{S}_{i}, S_{j} \in \mathcal{S}_{j}$ occurs in a solution to the Hybrid Problem, then there should be no edge $R\left(S_{i}, S_{j}\right)$. Let $\ell$ be the length- $k$ binary string, where the $i$ th and $j$ th bits are zeros and all other bits are ones. For each edge $R\left(S_{i}, S_{j}\right)$ on $S_{i} \in \mathcal{S}_{i}$ and $S_{j} \in \mathcal{S}_{j}$, we add an extra element $u_{S_{i} S_{j}}$ in $U_{\ell}$ and let $u_{S_{i} S_{j}} \in S_{i}, u_{S_{i} S_{j}} \in S_{j}$. Thus, $S_{i}^{\prime} \in \mathcal{S}_{i}$ and $S_{j}^{\prime} \in \mathcal{S}_{j}$ can both appear in the solution only when for all $u_{S_{i} S_{j}},\left(u_{S_{i} S_{j}} \notin S_{i}^{\prime}\right) \vee\left(u_{S_{i} S_{j}} \notin S_{j}^{\prime}\right)$, and this holds iff $R\left(S_{i}^{\prime}, S_{j}^{\prime}\right)=$ false.

- Case 2: If type $[i, j]=1$, then in a solution to the Hybrid Problem, $S_{i}$ and $S_{j}$ should have an edge $R\left(S_{i}, S_{j}\right)$ between them. Let $\ell$ be the length- $k$ binary string, where the $j$ th bit is zero and all other bits are ones. For each $S_{j} \in \mathcal{S}_{j}$, we add an extra element $u_{S_{j}}$ in $U_{\ell}$ and let $u_{S_{j}} \in S_{j}$. For each edge $R\left(S_{i}, S_{j}\right)$, we let $u_{S_{j}} \in S_{i}$. Thus, $S_{i}^{\prime} \in \mathcal{S}_{i}$ and $S_{j}^{\prime} \in \mathcal{S}_{j}$ can both appear in the solution only when for all $u_{S_{j}},\left(u_{S_{j}} \notin S_{j}^{\prime}\right) \vee\left(u_{S_{j}} \in S_{i}^{\prime}\right)$, and it holds iff $R\left(S_{i}^{\prime}, S_{j}^{\prime}\right)=$ true. 
After the above construction, we can drop the constraint (B) of the Hybrid Problem. We will ignore the relation $R$ and type in the Hybrid Problem. The problem now is to decide whether there exists tuple $\left(S_{1}, \ldots, S_{k}\right)$ being a solution to all $2^{k}$ Basic Problems. Then we can use the reductions in Lemma 5.1 to reduce all these Basic Problems to $B P\left[0^{k}\right]$, and then combine the $2^{k}$ instances to a large $B P\left[0^{k}\right]$ instance. Because the reductions do not change the solutions $S_{1}, \ldots, S_{k}$, there exists a solution to the large $B P\left[0^{k}\right]$ instance iff there exists a solution simultaneously to all the Basic Problem instances. Let $U_{\ell}{ }^{\prime}$ be the sub-universe of the $B P\left[0^{k}\right]$ instance reduced from the $B P[\ell]$ sub-problem. $\left(S_{1}, \ldots, S_{k}\right)$ is a solution to all Basic Problems iff their intersection is empty on every sub-universe $U_{\ell}^{\prime}$, iff their intersection is empty on universe $\bigcup_{\ell \in\{0,1\}^{k}} U_{\ell}^{\prime}$, i.e., it is a solution of a $B P\left[0^{k}\right]$ instance.

Multiplying the error probability in the reductions between Basic Problems by $2^{k}$, which is a constant number, and then taking a union bound, we get similar bounds of error probability for the Hybrid Problem.

5.2.2 Turing Reduction from General MC $\left(\exists^{k} \forall\right)$ Problems to the Hybrid Problem. The following lemma provides the last piece of the proof that sparse $k$-OV is complete for $M C\left(\exists^{k} \forall\right)$ under finegrained Turing reductions. The result follows by combining this lemma with Lemma 5.5.

LEMMA 5.6. For any integer $k \geq 2$, any problem in $M C\left(\exists^{k} \forall\right)$ is linear-time Turing reducible to the Hybrid Problem, namely, $\left(M C\left(\exists^{k} \forall\right), T(m)\right) \leq_{E C}($ Hybrid Problem, $T(O(m)))$.

Consider the problem $M C_{\varphi}$ where $\varphi=\left(\exists x_{1}\right) \ldots\left(\exists x_{k}\right)\left(\forall x_{k+1}\right) \psi\left(x_{1}, \ldots, x_{k+1}\right)$. An input graph $G$ can be preprocessed in linear time to ensure that it is a $(k+1)$-partite graph on vertices $V=$ $\left(V_{1}, \ldots, V_{k+1}\right)$, for example by creating $k+1$ copies of original vertex set.

W.l.o.g, we assume that for each occurrence of binary predicate $R_{t}\left(x_{i}, x_{j}\right), i \leq j$. Let $P_{k+1}$ be the set of unary and binary predicates in $\psi$ that involve variable $x_{k+1}$, and let $P_{\overline{k+1}}$ denote the set of the other predicates not including $x_{k+1}$. A partial interpretation $\alpha$ for $P_{\overline{k+1}}$ is a binary string of length $\left|P_{\overline{k+1}}\right|$, that encodes the truth values assigned to all predicates in $P_{\overline{k+1}}$. For each $i$ s.t. $1 \leq i \leq\left|P_{\overline{k+1}}\right|$, if the $i$ th predicate in $P_{\overline{k+1}}$ is assigned to true, then we set the $i$ th bit of $\alpha$ to one, otherwise, we set it to zero. For a tuple $\left(v_{1}, \ldots, v_{k}\right)$, we say it implies $\alpha$ (denoted by $\left(v_{1}, \ldots, v_{k}\right) \mid=\alpha$ ) iff when $\left(x_{1} \leftarrow v_{1}, \ldots, x_{k} \leftarrow v_{k}\right)$, the evaluations of all predicates in $P_{\overline{k+1}}$ are the same as the values specified by $\alpha$.

For each $\alpha \in\{0,1\}^{P_{\overline{k+1}}}$, we create a distinct Hybrid Problem instance $H_{\alpha}$. If any of the Hybrid Problems accepts, then we accept. Let $\left.\psi\right|_{\alpha}\left(x_{1}, \ldots, x_{k+1}\right)$ be $\psi$ after replacing all occurrences of predicates in $P_{\overline{k+1}}$ by their corresponding truth values specified by $\alpha$. The following steps show how to create $H_{\alpha}$ from $\alpha$ and $\left.\psi\right|_{\alpha}\left(x_{1}, \ldots, x_{k+1}\right)$.

Step 1: Construction of sets. We introduce colors, which are partial interpretations defined on some specific subsets of the predicates concerning variable $x_{k+1}$. We call them "colors" because they can be considered as a kind of labels on $\left(v_{i}, v_{k+1}\right)$ pairs. For each $i \in\{1, \ldots, k\}$, we give all the unary predicated defined on $x_{i}$ and binary predicates defined on $\left(x_{i}, x_{k+1}\right)$ (including those on $\left.\left(x_{k+1}, x_{i}\right)\right)$ a canonical order. We use $P_{i}$ to denote the set of these predicates for each $i$. Let a color be a partial interpretation for $P_{i}$, which is a binary string of length $\left|P_{i}\right|$, encoding the truth values assigned to all predicates in $P_{i}$. For each $j$ s.t. $1 \leq j \leq\left|P_{i}\right|$, if the $j$ th predicate in $P_{i}$ is assigned to true, then we set the $j$ th bit of the color to one, otherwise, we set it to zero. For a color $\left.c_{i} \in\{0,1\}\right\}_{i}^{\left|P_{i}\right|}$, we say $\left(v_{i}, v_{k+1}\right) \models c_{i}$ iff when $x_{i} \leftarrow v_{i}$ and $x_{k+1} \leftarrow v_{k+1}$, the values of all predicates in $P_{i}$ are the same as the corresponding bits of $c_{i}$. We refer to the colors where all bits are zeros as the background colors. These colors are special because they correspond to interpretations where all predicates in 
$P_{i}$ are false, i.e., we cannot directly go through all pairs $\left(v_{i}, v_{k+1}\right)$, where $\left(v_{i}, v_{k+1}\right) \mid=0^{\left|P_{i}\right|}$, since this is a co-sparse relation. So we need to deal with these pairs separately.

For a vertex combination $\left(v_{1}, \ldots, v_{k+1}\right)$, where $\left(v_{i}, v_{k+1}\right) \models c_{i}$ on all $1 \leq i \leq k$, the $k$-color-tuple $\left(c_{1}, \ldots, c_{k}\right)$ forms a color combination, which corresponds to truth values assigned to all the predicates in $P_{k+1}$.

For each $v_{i} \in V_{i}$, where $1 \leq i \leq k$, we create set $S_{v_{i}}$ in the set family $\mathcal{S}_{i}$. For each $v_{k+1} \in V_{k+1}$, and each color combination $\left(c_{1}, \ldots, c_{k}\right)$ s.t. $c_{i} \in\{0,1\}^{\left|P_{i}\right|}$ and the values of all predicates specified by $\left(c_{1}, \ldots, c_{k}\right)$ make $\left.\psi\right|_{\alpha}$ evaluate to false (in which case we say $\left(c_{1}, \ldots, c_{k}\right)$ does not satisfy $\left.\psi\right|_{\alpha}$ ), we create an element $u_{\left(v_{k+1}, c_{1}, \ldots, c_{k}\right)}$ in $U$. We call a string $C \in\{0,1\}^{k}$ an encoding of a color combination $\left(c_{1}, \ldots, c_{k}\right)$ when on all indices $i \in\{1, \ldots, k\}, C[i]=1$ iff $c_{i}=0^{\left|P_{i}\right|}$. We put each element $u_{\left(v_{k+1}, c_{1}, \ldots, c_{k}\right)}$ in the sub-universe $U_{C}$ iff $C$ is an encoding of $\left(c_{1}, \ldots, c_{k}\right)$.

Next we will construct the sets. For each $v_{i} \in V_{i}$, let $S_{v_{i}}$ be

$$
\begin{aligned}
& S_{v_{i}}=\left\{u_{\left(v_{k+1}, c_{1}, \ldots, c_{k}\right)} \mid\left(c_{1}, \ldots, c_{k}\right) \text { does not satisfy }\left.\psi\right|_{\alpha},\right. \text { and } \\
& \left.\quad\left(\left(c_{i} \neq 0^{\left|P_{i}\right|},\left(v_{i}, v_{k+1}\right) \vDash c_{i}\right), \text { or }\left(c_{i}=0^{\left|P_{i}\right|},\left(v_{i}, v_{k+1}\right) \mid \vDash c_{i}=0^{\left|P_{i}\right|}\right)\right)\right\} .
\end{aligned}
$$

To construct such sets, for each edge on $\left(x_{i}, x_{k+1}\right)$ (and $\left(x_{k+1}, x_{i}\right)$ ), we do the following. Assume the current vertex pair is $\left(v_{i}, v_{k+1}\right)$.

(1) First, let set $S_{v_{i}}$ contain all elements $u_{\left(v_{k+1}, c_{1}, \ldots, c_{k}\right)}$ in $U$, where $c_{i}$ is a fixed color such that $\left(v_{i}, v_{k+1}\right) \mid=c_{i}$, and the other colors $c_{j}$ can be any string in $\{0,1\}^{\left|P_{j}\right|}$.

(2) Next, let set $S_{v_{i}}$ contain all elements $u_{\left(v_{k+1}, c_{1}, \ldots, c_{k}\right)}$ in $U$, where $c_{i}=0^{\left|P_{i}\right|}$ (here $\left(v_{i}, v_{k+1}\right) \not \models$ $c_{i}=0^{\left|P_{i}\right|}$ because there is some edge connecting $v_{i}$ and $v_{k+1}$, meaning at least one bit in $c_{i}$ is 1$)$, and the other colors $c_{j}$ can be any string in $\{0,1\}^{\left|P_{j}\right|}$.

In other words, in the sub-universe labeled by $0^{k}$, which is made up of elements $u_{\left(v_{k+1}, c_{1}, \ldots, c_{k}\right)}$ such that none of the $c_{i}$ equals $0^{\left|P_{i}\right|}$, and that $\left(c_{1}, \ldots, c_{k}\right)$ does not satisfy $\left.\psi\right|_{\alpha}$, a set $S_{v_{i}}$ contains an element $u_{\left(v_{k+1}, c_{1}, \ldots, c_{k}\right)}$ iff $\left(v_{i}, v_{k+1}\right) \mid=c_{i}$. However, in any sub-universe labeled by $C$, where the $i$ th bit of $C$ is 1 , i.e., those are made up of elements $u_{\left(v_{k+1}, c_{1}, \ldots, c_{k}\right)}$ such that $c_{i}=0^{\left|P_{i}\right|}$ and that $\left(c_{1}, \ldots, c_{k}\right)$ does not satisfy $\left.\psi\right|_{\alpha}$, a set $S_{v_{i}}$ contains an element $u_{\left(v_{k+1}, c_{1}, \ldots, c_{k}\right)}$ iff $\left(v_{i}, v_{k+1}\right) \not c_{i}=$ $0^{\left|P_{i}\right|}$.

Analysis. Now we show the above construction achieves constraint (A) in the definition of the Hybrid Problem.

- Assume that $\left(v_{1}, \ldots, v_{k}\right)$ does not satisfy $\left.\left(\forall v_{k+1}\right) \psi\right|_{\alpha}\left(x_{1}, \ldots, x_{k+1}\right)$, i.e., there exists some $v_{k+1} \in V_{k+1}$ such that $\left.\psi\right|_{\alpha}\left(v_{1}, \ldots, v_{k+1}\right)$ is false. Then consider the specific color combination $\left(c_{1}, \ldots, c_{k}\right)$, where on each $i,\left(v_{i}, v_{k+1}\right) \mid=c_{i}$. So $\left(c_{1}, \ldots, c_{k}\right)$ does not satisfy $\left.\psi\right|_{\alpha}\left(x_{1}, \ldots, x_{k+1}\right)$. Thus there exists an element $u_{\left(v_{k+1}, c_{1}, \ldots, c_{k}\right)}$ in $U$.

If none of the colors in combination $\left(c_{1}, \ldots, c_{k}\right)$ is the background color, then the encoding of $\left(c_{1}, \ldots, c_{k}\right)$ is the string $0^{k}$. Thus, the element $u_{\left(v_{k+1}, c_{1}, \ldots, c_{k}\right)}$ is in sub-universe $U_{0^{k}}$. By our construction, $u_{\left(v_{k+1}, c_{1}, \ldots, c_{k}\right)}$ is contained in all of $S_{v_{1}}, \ldots, S_{v_{k}}$, as shown on the left side of Figure 5. This is because when we went through all the edges, at the edge between $\left(v_{i}, v_{k+1}\right)$, we put $u_{\left(v_{k+1}, c_{1}, \ldots, c_{k}\right)}$ in $S_{v_{i}}$, since none of the colors is background. Thus $\left(\exists u \in U_{0^{k}}\right)\left[\bigwedge_{i=1}^{k}\left(u \in S_{v_{i}}\right)\right]$, so it is not the case that $\left(\forall u \in U_{0^{k}}\right)\left[\bigvee_{i=1}^{k} \neg\left(u \in S_{v_{i}}\right)\right]$, which means $S_{v_{1}}, \ldots, S_{v_{k}}$ is not a solution of $B P\left[0^{k}\right]$ on sub-universe $U_{0^{k}}$.

If some of the colors $c_{i}$ in the color combination $\left(c_{1}, \ldots, c_{k}\right)$ equal the background color $0^{\left|P_{i}\right|}$, then in the encoding $C$ of $\left(c_{1}, \ldots, c_{k}\right), C[i]=1$. Thus, the element $u_{\left(v_{k+1}, c_{1}, \ldots, c_{k}\right)}$ is in the sub-universe $U_{C}$. By our construction, $u_{\left(v_{k+1}, c_{1}, \ldots, c_{k}\right)}$ is contained in sets $S_{v_{i}}$ for all 

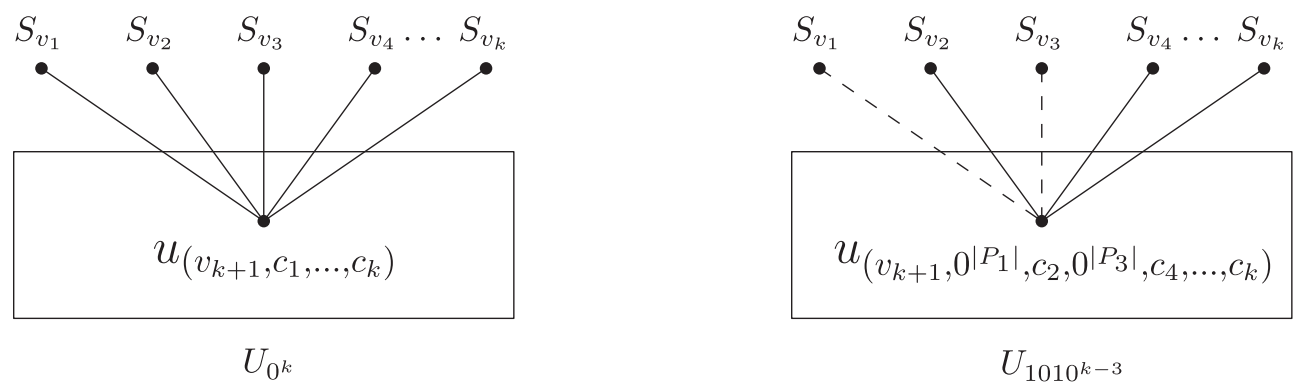

Fig. 5. The formula is satisfied iff there exists $\left(S_{v_{1}}, S_{v_{2}}, \ldots, S_{v_{k}}\right)$ so that there does not exist such an element $u$ in any of the sub-universes: The left figure illustrates the case where none of $c_{1}, \ldots, c_{k}$ is a background color. The right is the case where only $c_{1}$ and $c_{3}$ are background colors. (The dashed lines stand for nonexisting edges.)

indices $i$, where $c_{i}$ is not the background color $0^{\left|P_{i}\right|}$, and is not contained in sets $S_{v_{j}}$ for all indices $j$, where $c_{j}$ is the background color $0^{\left|P_{j}\right|}$. The latter case is because for each index $j$, where $c_{j}$ is the background color, there is no edge connecting the pair of vertices $\left(v_{j}, v_{k+1}\right)$. So we did not put $u_{\left(v_{k+1}, c_{1}, \ldots, c_{k}\right)}$ in $S_{v_{j}}$.

(The right side of Figure 5 demonstrates the example where $c_{1}$ and $c_{3}$ are the background colors while other colors are not.)

Thus

$$
\left(\exists u \in U_{C}\right)\left[\bigwedge_{i \in\{1, \ldots, k\}, C[i]=0}\left(u \in S_{v_{i}}\right) \wedge \bigwedge_{i \in\{1, \ldots, k\}, C[i]=1}\left(\neg\left(u \in S_{v_{i}}\right)\right)\right],
$$

so it is not the case that

$$
\left(\forall u \in U_{C}\right)\left[\bigvee_{i \in\{1, \ldots, k\}, C[i]=0}\left(\neg\left(u \in S_{v_{i}}\right)\right) \vee \bigvee_{i \in\{1, \ldots, k\}, C[i]=1}\left(u \in S_{v_{i}}\right)\right],
$$

which means $S_{v_{1}}, \ldots, S_{v_{k}}$ is not a solution of $B P[C]$ on sub-universe $U_{C}$.

- However, assume that $\left(v_{1}, \ldots, v_{k}\right)$ satisfies $\left.\left(\forall v_{k+1}\right) \psi\right|_{\alpha}\left(v_{1}, \ldots, v_{k+1}\right)$. We claim that for all $\ell \in\{0,1\}^{k},\left(S_{v_{1}}, \ldots, S_{v_{k}}\right)$ is a solution to Basic Problem $B P[\ell]$.

Consider the sub-universe $U_{C}$ for each $C \in\{0,1\}^{k}$. If $C=0^{k}$, i.e., the sub-universe is $U_{0^{k}}$ corresponding to $B P\left[0^{k}\right]$, then none of the elements $u_{\left(v_{k+1}, c_{1}, \ldots, c_{k}\right)}$ in $U_{0^{k}}$ contains any background color among its $c_{1}, \ldots, c_{k}$. For the sake of contradiction, suppose there exists an element $u_{\left(v_{k+1}, c_{1}, \ldots, c_{k}\right)}$ that is contained in all sets $S_{v_{1}}, \ldots, S_{v_{k}}$. So by our construction of sets, for each $i \in\{1, \ldots, k\},\left(v_{i}, v_{k+1}\right) \mid=c_{i}$. Recall that the color combination $\left(c_{1}, \ldots, c_{k}\right)$ in any element $u_{\left(v_{k+1}, c_{1}, \ldots, c_{k}\right)}$ does not satisfy $\left.\psi\right|_{\alpha}$. Then this means the vertex $v_{k+1}$ does not satisfy $\left.\psi\right|_{\alpha}\left(v_{1}, \ldots, v_{k}, v_{k+1}\right)$, which leads to a contradiction.

Thus on $\left(S_{v_{1}}, \ldots, S_{v_{k}}\right)$, it is not the case that $\left(\exists u \in U_{0^{k}}\right)\left[\bigwedge_{i=1}^{k}\left(u \in S_{v_{i}}\right)\right]$, implying $\left(S_{v_{1}}, \ldots, S_{v_{k}}\right)$ satisfies $\left(\forall u \in U_{0^{k}}\right)\left[\bigvee_{i=1}^{k} \neg\left(u \in S_{v_{i}}\right)\right]$. So it is a solution of the Basic Problem $B P\left[0^{k}\right]$ on sub-universe $U_{0^{k}}$.

If $C \neq 0^{k}$, for the sake of contradiction, suppose there exists an element $u_{\left(v_{k+1}, c_{1}, \ldots, c_{k}\right)}$ such that among $S_{v_{1}}, \ldots, S_{v_{k}}$, then it is contained in set $S_{v_{i}}$ iff $C[i]=0$. Then by our construction of sets, this means for all $i$ such that $C[i]=0,\left(v_{i}, v_{k+1}\right) \models c_{i}$; while for all $i$ such that $C[i] \neq 0,\left(v_{i}, v_{k+1}\right)=0^{\left|P_{i}\right|}=c_{i}$. Combining the two statements, for all $i,\left(v_{i}, v_{k+1}\right) \mid=c_{i}$. Recall again that the color combination $\left(c_{1}, \ldots, c_{k}\right)$ in any element $u_{\left(v_{k+1}, c_{1}, \ldots, c_{k}\right)}$ does not 
satisfy $\left.\psi\right|_{\alpha}$. This implies the vertex $v_{k+1}$ does not satisfy $\left.\psi\right|_{\alpha}\left(v_{1}, \ldots, v_{k+1}\right)$, which leads to a contradiction.

Thus on $\left(S_{v_{1}}, \ldots, S_{v_{k}}\right)$, it is not the case that

$$
\left(\exists u \in U_{C}\right)\left[\bigwedge_{i \in\{1, \ldots, k\}, C[i]=0}\left(u \in S_{v_{i}}\right) \wedge \bigwedge_{i \in\{1, \ldots, k\}, C[i]=1}\left(\neg\left(u \in S_{v_{i}}\right)\right)\right],
$$

implying $\left(S_{v_{1}}, \ldots, S_{v_{k}}\right)$ satisfies

$$
\left(\forall u \in U_{C}\right)\left[\bigvee_{i \in\{1, \ldots, k\}, C[i]=0}\left(\neg\left(u \in S_{v_{i}}\right)\right) \vee \bigvee_{i \in\{1, \ldots, k\}, C[i]=1}\left(u \in S_{v_{i}}\right)\right] .
$$

So it is a solution of the Basic Problem $B P[C]$ on sub-universe $U_{C}$.

In summary, there exists a tuple $\left(v_{1}, \ldots, v_{k}\right)$ such that $\left.\left(\forall v_{k+1}\right) \psi\right|_{\alpha}\left(v_{1}, \ldots, v_{k}, v_{k+1}\right)$ holds true, iff there exist sets $\left(S_{v_{1}}, \ldots, S_{v_{k}}\right)$ such that for all $\ell \in\{0,1\}^{k},\left(S_{v_{1}}, \ldots, S_{v_{k}}\right)$ is a solution of Basic Problem $B P[\ell]$ on sub-universe $U_{\ell}$. Thus our reduction satisfies constraint (A) of the Hybrid Problem.

Step 2: Construction of $R$ and type. Next, we consider the predicates in $P_{\overline{k+1}}$, which are predicates unrelated to variable $x_{k+1}$. We create edges for predicate $R$ according to the current partial interpretation $\alpha$.

For a pair of vertices $v_{i} \in V_{i}$ and $v_{j} \in V_{j}$, where $1 \leq i<j \leq k$, we say $\left(v_{i}, v_{j}\right)$ agrees with $\alpha$ if the evaluations of all predicates on $\left(x_{i}, x_{j}\right)$ (including $\left.\left(x_{j}, x_{i}\right)\right)$ when $x_{i} \leftarrow v_{i}, x_{j} \leftarrow v_{j}$, equals the truth values of corresponding predicates specified by $\alpha$.

- Case 1: At least one predicate on $\left(x_{i}, x_{j}\right)$ in $\alpha$ is true. (i.e., $\left(x_{i}, x_{j}\right)$ is in a sparse relation) For all edges $\left(v_{i}, v_{j}\right)$ (including $\left(v_{j}, v_{i}\right)$ ), where $v_{i} \in V_{i}$ and $v_{j} \in V_{j}$ and $i<j \leq k$, if $\left(v_{i}, v_{j}\right)$ agrees with $\alpha$, then we create edge $R\left(S_{v_{i}}, S_{v_{j}}\right)$. Finally, we make type $[i, j]=1$ in the Hybrid Problem $H_{\alpha}$.

- Case 2: All predicates on $\left(x_{i}, x_{j}\right)$ in $\alpha$ are false. (i.e., $\left(x_{i}, x_{j}\right)$ is in a co-sparse relation) For all edges $\left(v_{i}, v_{j}\right)$ (including $\left(v_{j}, v_{i}\right)$ ), where $v_{i} \in V_{i}$ and $v_{j} \in V_{j}$ and $i<j \leq k$, if $\left(v_{i}, v_{j}\right)$ does not agree with $\alpha$, then we create edge $R\left(S_{v_{i}}, S_{v_{j}}\right)$. Finally, we make type $[i, j]=0$ in the Hybrid Problem $H_{\alpha}$.

Analysis. We prove that $\left(v_{i}, v_{j}\right)$ can appear in the solution of $H_{\alpha}$ only if when it agrees with $\alpha$. If $\left(v_{i}, v_{j}\right)$ does not agree with $\alpha$, then we should not let them be in any solution of $H_{\alpha}$. This is done by the relation $R$ and the string type.

Consider the two cases. If in $\alpha$ some predicates on $\left(x_{i}, x_{j}\right)$ are true (i.e., set of tuples that agree with $\alpha$ is sparse), then in any $\left(v_{i}, v_{j}\right)$ that agrees with $\alpha$, there must be an edge in $G$ connecting $v_{i}$ and $v_{j}$. So we can add an edge (defined by relation $R$ ) on the corresponding sets $S_{v_{i}}, S_{v_{j}}$ and require there must be such an edge in the solution (i.e., type being 1).

However, if all predicates on $\left(x_{i}, x_{j}\right)$ in $\alpha$ are false (i.e., set of tuples agreeing with $\alpha$ is co-sparse), then in any $\left(v_{i}, v_{j}\right)$ that agrees with $\alpha$, there should not be any edge connecting $v_{i}$ and $v_{j}$. In this case, we turn to consider the tuples $\left(v_{i}, v_{j}\right)$ that do not agree with $\alpha$ (which is a sparse relation, instead of co-sparse). We create edges on the corresponding sets $S_{v_{i}}, S_{v_{j}}$ and require there must not be such an edge in the solution (i.e., type being 0 ).

Therefore, a tuple $\left(v_{1}, \ldots, v_{k}\right)$ implies $\alpha$ iff for all $i, j \in\{1, \ldots, k\}, i<j$, the truth value of relation $R\left(S_{v_{i}}, S_{v_{j}}\right)$ equals whether type $[i, j]=1$. Thus our reduction satisfies constraint (B) of the Hybrid Problem. 
From the analyses of the two steps, we have justified that: there exists $\left(v_{1}, \ldots, v_{k}\right)$ so that $\left(v_{1}, \ldots, v_{k}\right) \mid=\alpha$, and $\left.\psi\right|_{\alpha}$ holds for all $v_{k+1} \in V_{k+1}$, iff there exists $\left(S_{v_{1}}, \ldots, S_{v_{k}}\right)$ being a solution to the Hybrid Problem $H_{\alpha}$. Thus, if for any $\alpha \in\{0,1\}^{P_{\overline{k+1}}}$, the Hybrid Problem $H_{\alpha}$ accepts, then there exists a solution $\left(v_{1}, \ldots, v_{k}\right)$ so that $\psi\left(v_{1}, \ldots, v_{k}, v_{k+1}\right)$ holds for all $v_{k+1} \in V_{k+1}$. Otherwise there does not exist such a solution. From the above argument, we have proved the following claim.

Claim 5.1. The two propositions are equivalent:

(1) $M C_{\varphi}$ has a solution $x_{1} \leftarrow v_{1}, \ldots, x_{k} \leftarrow v_{k}$ such that $\left(\forall v_{k+1} \in V_{k+1}\right) \psi\left(v_{1}, \ldots, v_{k+1}\right)$ is satisfied.

(2) There exists an $\alpha \in\{0,1\}^{P_{\overline{k+1}}}$ so that $\left(S_{v_{1}}, \ldots, S_{v_{k}}\right) \mid=\alpha$, and $S_{v_{1}}, \ldots, S_{v_{k}}$ is a solution to the Hybrid Problem $H_{\alpha}$.

The running time of the whole reduction process is linear in the total number of edges in the graph, because the number of predicates is constant. Thus Lemma 5.6 follows.

\section{DERANDOMIZATION}

We derandomize the reduction in Section 5 for the $k=2$ case, so that the whole proof of Theorems 1.2 and 1.4 is determistic. The derandomization of the randomized universe-shrinking selfreduction uses the technique of nearly disjoint sets similar to the construction of pseudorandom generator by Nisan and Widgerson in Reference [28].

In this section, for simplicity we use $S C(x)$ (respectively, $S D(x)$ ) to denote Set Containment, a.k.a. the Basic Problem $B P[01]$ (respectively, Set Disjointness, a.k.a. the Basic Problem [00] or Sparse OV) on universe of size $x$, and use HP for the Hybrid Problem.

Lemma 6.1. For any $2^{\Omega(\sqrt{\log n})} \leq s<m^{1 / 3}$, there is a deterministic universe-shrinking selfreduction for $S C$ such that $\left(S C(n), m^{2} / s\right) \leq_{E C}\left(S C\left(O\left(s^{2} \log ^{2} n / \log ^{2} s\right), m^{2} / s^{3} \frac{\log ^{2} n}{\log ^{2} s}\right)\right)$.

LEMmA 6.2. For any $2^{\Omega(\sqrt{\log n})} \leq s<m^{1 / 3}$, there is a deterministic universe-shrinking selfreduction for $S D$ such that $\left(S D(n), m^{2} / s\right) \leq_{E C}\left(S D\left(O\left(s^{2} \log n / \log s\right), m^{2} / s^{3} \frac{\log n}{\log s}\right)\right)$.

The following reduction from the Hybrid Problem to Set Disjointness implies the model checking for any $\exists \exists \forall$ sentences on sparse structures can be reduced to moderate-dimension SD, and then to OV.

Lemma 6.3. For any $2^{\Omega(\sqrt{\log n})} \leq s<m^{1 / 3}$, where $m$ is the input size to the Hybrid Problem, there is a deterministic reduction algorithm such that $\left(H P, m^{2} / s\right) \leq_{E C}\left(S D\left(O\left(s^{2} \log ^{2} n / \log ^{2} s\right), m^{2} / s^{7} \frac{\log ^{3} n}{\log ^{3} s}\right)\right)$.

\subsection{Proof of Lemma 6.1}

This section presents the derandomization of the universe-shrinking self-reduction in Sections 5.1.1 for the Basic Problem $B P[01]$ (and equivalently $B P[10]$ ), i.e., when the corresponding Basic Problem is the Set Containment problem.

Pick $\ell=O(\log n / \log s)$ and prime number $q=O(s \log n / \log s)$, so that $s \ell<q$ and $q^{\ell}>n$. By Bertrand's postulate and that PRIMES is in P, we can find such a $q$ in time $\tilde{O}(\log n / \log s)$.

First, we use the algorithm in Section 5.1.1 to decide if there is a solution containing a set of size at least $s$, which takes time $O\left(\mathrm{~m}^{2} / \mathrm{s}\right)$. So next we only consider sets of size smaller than $s$.

We create a new universe $U^{\prime}$ of size $q^{2}$. Let $U^{\prime}$ be $G F(q) \times G F(q)$. Let element $u$ in universe $U$ correspond to a unique polynomial $p_{u}$ over $G F(q)$ of degree $\ell$. The number of different polynomials is $q^{\ell}$. Since $q^{\ell}>n$, the number of different polynomials is greater than the number of elements of $U$. 
Let $h$ be a hash function so that each element in $U$ is mapped to a set $h(u)=\left\{\left\langle i, p_{u}(i)\right\rangle \mid i \in\right.$ $G F(q)\}$ of size $q$. For set $S \subseteq U$, define $h(S)=\bigcup_{u \in S} h(u)$. Finally, $\mathcal{S}_{1}^{\prime}=\left\{h(S) \mid S \in \mathcal{S}_{1}\right\}$, and $\mathcal{S}_{2}^{\prime}$ is constructed similarly. Then we decide the $S C\left(q^{2}\right)$ instance that takes $\mathcal{S}_{1}^{\prime}$ and $\mathcal{S}_{2}^{\prime}$ as input.

If $S_{1} \subseteq S_{2}$, then $h\left(S_{1}\right) \subseteq h\left(S_{2}\right)$, and the call to the $S C\left(q^{2}\right)$ instance returns true.

If $S_{1} \nsubseteq S_{2}$ for all sets, we need to show that for each element $u_{1} \in S_{1} \backslash S_{2},\left|h\left(u_{1}\right) \cap h\left(S_{2}\right)\right|<q$. Then because $\left|h\left(u_{1}\right)\right|=q$, some element in $h\left(u_{1}\right)$ is not in $h\left(S_{2}\right)$, therefore $h\left(S_{1}\right) \nsubseteq \not h\left(S_{2}\right)$. To show $\left|h\left(u_{1}\right) \cap h\left(S_{2}\right)\right|<q$, observe that for each element $u_{2} \in S_{2}$, the intersection $h\left(u_{1}\right) \cap h\left(u_{2}\right)$ has size at most $\ell$, the degree of polynomial $p_{u_{1}}-p_{u_{2}}$. There are at most $s$ elements in $S_{2}$, thus $\left|h\left(u_{1}\right) \cap h\left(S_{2}\right)\right| \leq$ $s \ell<q$.

Thus, there exist $S_{1} \subseteq S_{2}$ in the original instance iff there exist $h\left(S_{1}\right) \subseteq h\left(S_{2}\right)$ in the constructed instance.

The time to create the new set is $O(m q \ell)$, which is less than $O\left(m^{2} / s\right)$. And its size is $m^{\prime} \leq m q$. Thus, if we can solve it in time $O\left(\mathrm{~m}^{\prime 2} /\right.$ poly $\left.(s)\right)$, where $s<m^{\epsilon}$ for all $\epsilon>0$, we can solve it in time $O\left(m^{2} q^{2} / \operatorname{poly}(s)\right)=O\left(m^{2} / \operatorname{poly}(s)\right)$.

\subsection{Proof of Lemma 6.2}

First, we use the algorithm in Section 5.1.1 to decide if there is a solution containing a set of size at least $s$, which takes time $O\left(\mathrm{~m}^{2} / \mathrm{s}\right)$. So next we only consider sets of size smaller than $s$.

Let $\ell=O(\log n / \log s)$, and let $q$ be a prime $\geq s^{2} \ell$, thus $q=O\left(s^{2} \ell\right)=O\left(s^{2} \frac{\log n}{\log s}\right)$. So $q^{\ell}>n$. By Bertrand's postulate, we can find such a $q$ in time $O\left(s^{2} \frac{\log n}{\log s}\right)$. We create a universe $U^{\prime}$ of size $q$.

Each element $u$ of $U$, which is a string of length $\log n$, can be viewed as the encoding of a polynomial $p_{u}$ over $G F(q)$ of degree $\frac{\log n}{\log q} \leq \frac{\log n}{\log s}=\ell$.

Let $a$ be an element in group $G F(q)$. For each element $u$ in $U$, we let hash function $h_{a}(u)=p_{u}(a)$. For set $S \subseteq U$, define $h_{a}(S)=\bigcup_{u \in S}\left\{h_{a}(u)\right\}$. The algorithm in the outermost loop enumerates all elements $a \in G F(q)$. For each $a$, we compute $h_{a}(S)$ for all sets $S$ in the input. Then we decide if there are two disjoint sets in the new instance. The algorithm makes $q$ queries to $S D(q)$ instances of input size $m$, each taking time $T(m)=m^{2} / s^{3} \frac{\log n}{\log s}=m^{2} / s q$, the running time for moderate-dimension OV. The total time is $q T(m)=O\left(\mathrm{~m}^{2} / \mathrm{s}\right)$.

For each pair of different elements $u$ and $v$ in $U$, the number of elements $a$ in $G F(q)$ so that $p_{u}(a)=p_{v}(a)$ is at most their degree $l<\log n$. Suppose $S_{1} \in \mathcal{S}_{1}$ and $S_{2} \in \mathcal{S}_{2}$ are a pair of disjoint sets. $h_{a}\left(S_{1}\right)$ and $h_{a}\left(S_{2}\right)$ are disjoint if all pairs of their elements are mapped to different elements in $G F(q)$. The total number of possible collisions is at most $s^{2} \log n$. Because $q>s^{2} \log n$, there exists at least one element $a$ in $G F(q)$ so that all pairs of elements in $S_{1}$ and $S_{2}$ are mapped to different elements by $h_{a}$.

If there are no disjoint sets, then for each $S_{1} \in \mathcal{S}_{1}$ and $S_{2} \in \mathcal{S}_{2}, h\left(S_{1} \cap S_{2}\right) \subseteq h\left(S_{1}\right) \cap h\left(S_{2}\right)$, so $h\left(S_{1}\right)$ and $h\left(S_{2}\right)$ are not disjoint. Thus, for every $a \in G F(q)$, the call to the $S D(\log n)$ instance returns false.

\subsection{Hybrid Problem}

In this section, we combine the above two deterministic reductions to solve the Hybrid Problem, which yields a deterministic reduction for Theorem 1.2 and Theorem 1.4. Here we use a similar version of the Hybrid Problem as defined in Section 5.2 but without the relation $R$ and the string type. More formally, we consider the Hybrid Problem defined as follows:

Problem $H P$

Input: $\mathcal{S}_{1}, \mathcal{S}_{2}$, each a set family of sets $S_{i}=A_{i} \cup B_{i} \cup C_{i} \cup D_{i}$, where $A_{i}, B_{i}, C_{i}, D_{i}$ are subsets of disjoint universes $U_{A}, U_{B}, U_{C}, U_{D}$, respectively. 
Output: Whether there exist $S_{i} \in \mathcal{S}_{1}$ and $S_{j} \in \mathcal{S}_{2}$ so that

(1) $A_{i} \cap A_{j}=\emptyset$ (Set Disjointness)

(2) $B_{i} \subseteq B_{j}$ (Set Containment)

(3) $C_{i} \supseteq C_{j}$ (Set Containment reversed)

(4) $D_{i} \cup D_{j}=U_{D}(2$-Set Cover $)$

The results in Section 5.2 can be applied to this version of Hybrid Problem, so that the model checking for first-order sentences of form $\exists \exists \forall$ can be reduced to the Hybrid Problem. More precisely, $(M C(\exists \exists \forall), T(O(m))) \leq(H P, T(m))$.

Proof of Lemma 6.3. First, we decide if there is a solution containing a set of size at least $s$, as described in the previous subsections, using time $O\left(\mathrm{~m}^{2} / \mathrm{s}\right)$. So next we only consider sets of size smaller than $s$.

If $\left|U_{D}\right| \geq 2 s$, then for all pairs of $i, j, D_{i}$ and $D_{j}$ cannot cover $U_{D}$, so we return false. Otherwise for $i$ and all $j$, we create sets $U_{D} \backslash D_{i}$ and $U_{D} \backslash D_{j}$. So $D_{1} \cup D_{2}=U_{D}$ iff $\left(U_{D} \backslash D_{i}\right) \cap\left(U_{D} \backslash D_{j}\right)=\emptyset$. The resulting instance size is $O(m s)$.

Then, we use Lemma 6.1 self-reductions for Set Containment on the $B$ 's and $C$ 's, so the created sets $B_{i}^{\prime}, B_{j}^{\prime}$ and $C_{i}, C_{j}$ are on universes of size $O\left(s^{2} \frac{\log ^{2} n}{\log ^{2} s}\right)$. For each $j$, we create set $U_{B} \backslash B_{j}^{\prime}$, so $B_{i} \subseteq B_{j}$ iff $B_{i}^{\prime} \subseteq B_{j}^{\prime}$ iff $B_{i}^{\prime} \cap\left(U_{B} \backslash B_{j}^{\prime}\right)=\emptyset$. Similarly for each $i$ we create $U_{C} \backslash C_{i}^{\prime}$, so $C_{i} \supseteq C_{j}$ iff $C_{i}^{\prime} \supseteq C_{j}^{\prime}$ iff $\left(U_{C} \backslash C_{i}^{\prime}\right) \cap C_{j}^{\prime}=\emptyset$. The resulting instance size is $O\left(m \cdot s^{2} \frac{\log ^{2} n}{\log ^{2} s}\right)$.

Finally, we use Lemma 6.2 self-reductions for Set Disjointness on the original $A$ 's. So in each call to the oracle, the created sets $A_{i}^{\prime}, A_{j}^{\prime}$ are on universes of size $O\left(s^{2} \frac{\log n}{\log s}\right)$. For each $i$ and each $j$, we create sets $S_{i}^{\prime}=A_{i}^{\prime} \cup B_{i}^{\prime} \cup\left(U_{C} \backslash C_{i}^{\prime}\right) \cup\left(U_{D} \backslash D_{i}\right)$ and $S_{j}^{\prime}=A_{j}^{\prime} \cup\left(U_{B} \backslash B_{j}^{\prime}\right) \cup C_{j}^{\prime} \cup\left(U_{D} \backslash D_{j}\right)$. By the argument above, $S_{i}^{\prime} \cap S_{j}^{\prime}=\emptyset$ iff $A_{i}^{\prime} \cap A_{j}^{\prime}=\emptyset$ and $B_{i} \subseteq B_{j}$ and $C_{i} \supseteq C_{j}$ and $D_{i} \cup D_{j}=U_{D}$. If $A_{i} \cap$ $A_{j}=\emptyset$, then in at least one call to the oracle $A_{i}^{\prime} \cap A_{j}^{\prime}=\emptyset$ and thus the call will return true as long as the conditions on $B, C, D$ 's are satisfied. If $A_{i} \cap A_{j} \neq \emptyset$, then all calls return false.

The size of the new instance is $O\left(m \cdot s^{2} \frac{\log ^{2} n}{\log ^{2} s}\right)$. In the reduction, we make $s^{2} \frac{\log n}{\log d}$ calls to the algorithm for Set Disjointness on small universe. Thus if $S D\left(O\left(s^{2} \frac{\log ^{2} n}{\log ^{2} s}\right)\right)$ has algorithms in time $O\left(m^{2} / s^{7} \frac{\log ^{5} n}{\log ^{5} s}\right)$, we get running time $s^{2} \frac{\log n}{\log d} \cdot O\left(\left(m \cdot s^{2} \frac{\log ^{2} n}{\log ^{2} s}\right)^{2} / s^{7} \frac{\log ^{5} n}{\log ^{5} s}\right)=O\left(m^{2} / s\right)$.

This gives a reduction from the general first-order model checking problems to the Hybrid Problem.

\subsection{Extending to More Quantifiers}

The derandomization can be extended to quantifiers $k+1$ for integer $k \geq 2$. The reduction combines the reductions for Set Containment and Set Disjointness.

Recall from Section 5.1, a Basic Problem $B P[\ell]$, where $\ell \neq 1^{k}$ can be considered as deciding $\left(\exists S_{1}\right) \ldots\left(\exists S_{k}\right)(\forall u)\left[\left(\bigvee_{j=1}^{i}\left(u \notin S_{j}\right)\right) \vee\left(\bigvee_{j=i+1}^{k}\left(u \in S_{j}\right)\right)\right]$, or equivalently $\left(\exists S_{1}\right) \ldots\left(\exists S_{k}\right)$

$\left[\left(\cap_{j=1}^{i} S_{j}\right) \subseteq\left(\bigcup_{j=i+1}^{k} S_{j}\right)\right]$. for some $i$ such that $0 \leq i \leq k$. Again, we map each element in $U$ to a set of elements in a small universe $U^{\prime}$ by some function $h$, and thus map each set $S$ in $U$ to a set $h(S)$ in $U^{\prime}$.

Let $q_{1}, q_{2}$ be the $q$ defined in Sections 6.1 and 6.2, respectively. Here $q_{2}$ is a prime number larger than $s^{k-i} \ell$. For each element $u$, for each element $a \in G F\left(q_{2}\right)$ we map it to a set of tuples $h(u)=\left\{\left\langle u_{S C}, u_{S D}\right\rangle \mid u_{S C} \in h^{S C}(u), u_{S D} \in h_{a}^{S D}(u)\right\}$, where $h^{S C}$ and $h_{a}^{S D}$ are the functions $h$ and $h_{a}$ defined in Sections 6.1 and 6.2, respectively, and then we make a query for the $B P[\ell]$ instance created from the mapping $h$. Thus we make $q_{2}$ queries in all, and accept if at least one of the queries is accepted. 
If there exist sets $S_{1}, \ldots, S_{k}$ such that $\bigcap_{j=1}^{i} S_{j} \subseteq \bigcup_{j=i+1}^{k} S_{j}$, by generalizing the analysis in Section 6.2, in at least one query, then the set $\bigcap_{j=1}^{i} h\left(S_{j}\right)$ does not contain any element not in $h\left(\bigcap_{j=1}^{i} S_{j}\right)$. And by generalizing the analysis in Section 6.1, in each query, the set $\bigcup_{j=i+1}^{k} h\left(S_{j}\right)=$ $h\left(\bigcap_{j=1}^{i} S_{j}\right)$ is always contained in $h\left(\bigcup_{j=i+1}^{k} S_{j}\right)$ that is contained in $\bigcup_{j=i+1}^{k} h\left(S_{j}\right)$. So we get the following reduction: $\left(B P[\ell](n), m^{k} / s\right) \leq_{E C}\left(B P[\ell](\operatorname{poly}(s)), m^{k} / \operatorname{poly}(s)\right)$.

\section{EXTENDING ALGORITHMS AND HARDNESS RESULTS TO HYPERGRAPHS}

This section gives a reduction from $M C(\exists \exists \forall)$, i.e., the model checking for $\exists \exists \forall$ formulas on hypergraphs, to the model checking for $\exists \exists \forall$ formulas on graphs, where there are only unary and binary relations. We will prove the following lemma.

LEMMA 7.1. If $M C\left(\exists^{k} \forall\right)$ on graphs is solvable in time $T(m)$, then $M C\left(\exists^{k} \forall\right)$ on hypergraphs is solvable in $T(O(m))+O\left(m^{k-1 / 2}\right)$.

For a three-quantifier formula $(\exists x)(\exists y)(\forall z) \psi(x, y, z)$, where $x \in X, y \in Y, z \in Z$, we prove that it can be decided in time $O\left(\mathrm{~m}^{3 / 2}+T(O(m))\right)$, where $T$ is the running time for the model checking of three-quantifier formulas on graphs.

Let relation $N(x, y)$ be the edges of the Gaifman graph, which means $N(x, y)=$ true iff there exists some $z$ such that there is a hyperedge $R_{i}(x, y, z)=$ true (the order of $x, y, z$ can be interchanged). Note that each tuple in the relations contributes to only constantly many tuples of $N$. So $|N|=O(m)$, and we can construct $N$ in linear time.

Let $\psi(x, y, z)$ be a quantifier-free formula. We define $\psi^{*}(x, y, z)$ be $\psi(x, y, z)$, where all occurrences of ternary predicates are replaced by false. Thus, it contains only unary and binary predicates. Formula $(\exists x)(\exists y)(\forall z) \psi(x, y, z)$ is equivalent to $(\exists x)(\exists y)(\forall z)[N(x, y) \wedge \psi(x, y, z)] \vee$ $(\exists x)(\exists y)(\forall z)\left[\neg N(x, y) \wedge \psi^{*}(x, y, z)\right]$.

We can decide $(\exists x)(\exists y)(\forall z)\left[\neg N(x, y) \wedge \psi^{*}(x, y, z)\right]$ using the algorithm for graphs, because all relations are binary. To decide $(\exists x)(\exists y)(\forall z)[N(x, y) \wedge \psi(x, y, z)]$, we consider three types of $x$ 's and $y$ 's. Let $\operatorname{deg}(x)$ be the degree of $x$ in the Gaifman graph.

- Type 1: $\operatorname{deg}(x) \geq \sqrt{\boldsymbol{m}}$. It is similar to deciding "large sets" for Basic Problems in Section 5.1.1. In the outer loop, enumerate all such $x$ 's. For each $x$, we modify the model checking problem to an instance of $M C(2)$, by treating $x$ as a constant. The number of such $x$ 's is at most $O(m / \sqrt{m})=O(\sqrt{m})$, and deciding an $M C(2)$ problem runs in time $O(m)$. So the total running time is $O(\sqrt{m} \cdot m)=O\left(m^{3 / 2}\right)$.

- Type 2: $\operatorname{deg}(y) \geq \sqrt{\boldsymbol{m}}$. Use the same method as above by exchanging the order of $x$ and $y$. The running time is also $O\left(\mathrm{~m}^{3 / 2}\right)$.

- Type 3: $\operatorname{deg}(x)<\sqrt{m}$ and $\operatorname{deg}(y)<\sqrt{m}$. Enumerate all pairs of such $x$ 's and $y$ 's. Then in the inner loop, we enumerate all their neighbors in $Z$. In this way, for each $z \in Z$ such that $z$ is a neighbor of $x$ or $y$, we can categorize it by the truth value of all predicates. For all other $z$ 's, we know all the predicates are false. Thus we can decide if all $z \in Z$ satisfy $\psi$. Because all these $x$ 's and $y$ 's are adjacent, the time for enumerating pairs of $x$ and $y$ is $O(m)$, and the time for enumerating all their neighbors in $Z$ is $O(\sqrt{m})$. So the total running time is $O(\sqrt{m} \cdot m)=O\left(m^{3 / 2}\right)$.

Thus, for each pair $(x, y)$, where $N(x, y)=$ true, we can decide the model checking for $(\forall z) \psi(x, y, z)$ in time $O\left(m^{3 / 2}\right)$. For each pair $(x, y)$, where $N(x, y)=$ false, $(\forall z) \psi(x, y, z)$ is true iff $(\forall z)\left[\neg N(x, y) \wedge \psi^{*}(x, y, z)\right]$.

Similarly, for $M C\left(\exists^{k} \forall\right)$ problems where $\varphi=\left(\exists x_{1}\right) \ldots\left(\exists x_{k}\right)\left(\forall x_{k+1}\right) \psi\left(x_{1}, \ldots, x_{k+1}\right)$, we still consider the cases whether there exist some hyperedge between any pair of $x_{i}, x_{j}$, where $i, j \leq k$. We 
define relation $N\left(x_{i}, x_{j}\right)=$ true iff there exists some $x_{k}$ such that there is some hyperedge containing vertices $x_{i}, x_{j}$. We also define $\psi^{*}\left(x_{1}, \ldots, x_{k+1}\right)$ be $\psi\left(x_{1}, \ldots, x_{k+1}\right)$, where all occurrences of predicates with arities greater than two are replaced by false. So

$$
\begin{aligned}
\varphi= & \left(\exists x_{1}\right) \ldots\left(\exists x_{k}\right)\left(\forall x_{k+1}\right)\left[\bigvee_{i, j \in\{1, \ldots, k\}, i \neq j}\left(N\left(x_{i}, x_{j}\right) \wedge \psi\left(x_{1}, \ldots, x_{k+1}\right)\right]\right. \\
& \vee\left[\left(\bigwedge_{i, j \in\{1, \ldots, k\}, i \neq j} \neg N\left(x_{i}, x_{j}\right)\right) \wedge \psi^{*}\left(x_{1}, \ldots, x_{k+1}\right)\right] \\
= & \bigvee_{i, j \in\{1, \ldots, k\}, i \neq j}\left[\left(\exists x_{1}\right) \ldots\left(\exists x_{k}\right)\left(\forall x_{k+1}\right)\left[N\left(x_{i}, x_{j}\right) \wedge \psi\left(x_{1}, \ldots, x_{k+1}\right)\right]\right] \\
& \vee\left(\exists x_{1}\right) \ldots\left(\exists x_{k}\right)\left(\forall x_{k+1}\right)\left[\left(\bigwedge_{i, j \in\{1, \ldots, k\}, i \neq j} \neg N\left(x_{i}, x_{j}\right)\right) \wedge \psi^{*}\left(x_{1}, \ldots, x_{k+1}\right)\right] .
\end{aligned}
$$

To decide $\left(\exists x_{1}\right) \ldots\left(\exists x_{k}\right)\left(\forall x_{k+1}\right)\left[N\left(x_{i}, x_{j}\right) \wedge \psi\left(x_{1}, \ldots, x_{k+1}\right)\right]$, we do exhaustive search on the $k-2$ variables other than $x_{i}$ and $x_{j}$ (which in essence is a quantifier-eliminating downward reduction), which takes a factor of $O\left(m^{k-2}\right)$ in the running time. Then we process the variables $x_{i}$, $x_{j}, x_{k}$ in the same way as variables $x, y, z$ in the three-quantifier problem, that takes time $O\left(\mathrm{~m}^{3 / 2}\right)$. The total running time is $O\left(m^{k-1 / 2}\right)$.

To decide $\left(\exists x_{1}\right) \ldots\left(\exists x_{k}\right)\left(\forall x_{k+1}\right)\left[\left(\bigwedge_{i, j \in\{1, \ldots, k\}, i \neq j} \neg N\left(x_{i}, x_{j}\right)\right) \wedge \psi^{*}\left(x_{1}, \ldots, x_{k+1}\right)\right]$, we can use the algorithm for $M C\left(\exists^{k} \forall\right)$ problems on graphs, because the new formula has only unary and binary relations.

\section{HARDNESS OF $k$-OV FOR $M C\left(\forall \exists^{K-1} \forall\right)$}

In this section, we present an exact complexity reduction from any $M C\left(\forall \exists^{k-1} \forall\right)$ problem to a $M C\left(\exists^{k} \forall\right)$ problem, establishing the hardness of $k$-OV for these problems. This reduction gives an extension of the reduction from Hitting Set to Orthogonal Vectors in Reference [4] to sparse structures.

Lemma 8.1. For $k \geq 2$ and $s(m)$ a non-decreasing function such that $2^{\Omega(\sqrt{\log m})} \leq s(m)<m^{1 / 5}$, let $\varphi^{\prime}=\left(\exists x_{2}\right) \ldots\left(\exists x_{k}\right)\left(\forall x_{k+1}\right) \psi\left(x_{1}, \ldots, x_{k+1}\right)$. There is an exact complexity reduction

$$
\left(M C_{\left(\forall x_{1}\right) \varphi^{\prime}}, \frac{m^{k}}{s(\sqrt{m})}\right) \leq_{E C}\left(M C_{\left(\exists x_{1}\right) \varphi^{\prime}}, \frac{m^{k}}{s(m)}\right) .
$$

First, we show that in problem $M C_{\left(\exists x_{1}\right) \varphi^{\prime}}$, if graph $G$ satisfies $\left(\exists x_{1}\right) \varphi^{\prime}$, then we can find a satisfying value $v_{1}$ for variable $x_{1}$ by binary search. We divide the set $V_{1}$ into two halves, take each half of $V_{1}$ and query whether $\left(\exists x_{1}\right) \varphi^{\prime}$ holds true on the graph induced by this half of $V_{1}$ together with the original sets $V_{2}, \ldots, V_{k+1}$. If any half of $V_{1}$ works, then we can shrink the set of candidate values for $x_{1}$ by a half, and then recursively query again, until there is only one vertex $v_{1}$ left. So it takes $O\left(\log \left|V_{1}\right|\right)$ calls to find a $v_{1}$ in some solution. This means as long as there is a solution for $M C_{\exists x_{1} \varphi^{\prime}}$, we can find a satisfying $v_{1}$ efficiently, with $O(\log m)$ queries to the decision problem.

Step 1: Large degree vertices. Let $t=m^{(k-1) / k}$. We deal with vertices in $V_{1} \ldots V_{k}$ with degree greater than $t$. There are at most $m / t=m^{1 / k}$ such vertices. After pre-computing the sizes of all the sets, these large sets can be listed in time $O\left(m^{1 / k}\right)$.

- Step 1-1: Large degree vertices in $V_{1}$. For each vertex $v_{1} \in V_{1}$ with degree at least $t$, we create a formula $\psi_{v_{1}}$ on variables $x_{2}, \ldots, x_{k+1}$ from formula $\psi$, by replacing occurrences of 
unary predicates in $\psi$ on $x_{1}$ by constants, and replacing occurrences of binary predicates involving $x_{1}$ by unary predicates on the other variables. Then we check if the graph induced by $V_{2}, \ldots, V_{k+1}$ satisfies $\left(\exists x_{2}\right) \ldots\left(\exists x_{k}\right)\left(\forall x_{k+1}\right) \psi_{v_{1}}\left(x_{2}, \ldots, x_{k+1}\right)$ by running the baseline algorithm in time $O\left(\mathrm{~m}^{k-1}\right)$. If the new formula is satisfied, then we mark $v_{1}$ as "good." The total time complexity is $O\left(m^{1 / k}\right) \cdot O\left(m^{k-1}\right)=O\left(m^{k-1+1 / k}\right)$.

- Step 1-2: Large degree vertices in $V_{2}, \ldots, V_{k}$. Now we exhaustively search over all vertices $v_{1} \in V_{1}$ with degree less than $t$ in the outermost loop. For each such $v_{1}$, we find out all vertices $v_{i} \in V_{i}$ for $2 \leq i \leq k$, with degree at least $t$. Again, there are at most $O\left(m^{1 / k}\right)$ of them.

- Case 1: $k>2$. Because variables $x_{2}$ through $x_{k}$ are all quantified by $\exists$, we interchange their order so that the variable $x_{i}$ becomes the second-outermost variable $x_{2}$ (and thus the current $v_{i}$ becomes $v_{2}$ ). Next, for each $v_{1}$ and $v_{2}$ we construct a new formula $\psi_{\left(v_{1}, v_{2}\right)}$ on variables $x_{3}, \ldots, x_{k+1}$, by regarding $x_{1}$ and $x_{2}$ as fixed values $v_{1}$ and $v_{2}$, and then modify $\psi$ into $\psi_{\left(v_{1}, v_{2}\right)}$ similarly to the previous step. Again, we run the baseline algorithm to check whether the graph induced by the current $V_{3}, \ldots, V_{k+1}$ satisfies $\left(\exists x_{3}\right) \ldots\left(\exists x_{k+1}\right) \psi_{\left(v_{1}, v_{2}\right)}\left(x_{3}, \ldots, x_{k+1}\right)$, using time $O\left(m^{k-2}\right)$. If the formula is satisfied, then we mark the current $v_{1}$ as "good." The total time complexity is $O\left(\mathrm{~m} \cdot \mathrm{m}^{1 / k}\right)$. $\left(m^{k-2}\right)=O\left(m^{k-1+1 / k}\right)$.

- Case 2: $\boldsymbol{k}=2$. For each vertex $v_{2}$, we mark all the $v_{1}$ 's satisfying $\forall x_{3} \psi\left(v_{1}, v_{2}, x_{3}\right)$ as "good". This can be done in $O(m)$ using the algorithm for the base case of the baseline algorithm, by treating the current $v_{2}$ as constant. So this process runs in time $O\left(\mathrm{~m}^{1 / k}\right)$. $O(m)=O\left(m^{3 / 2}\right)$.

If not all vertices in $V_{1}$ with degree at least $t$ are marked "good," then we reject. Otherwise, go to Step 2.

Step 2: Small degree vertices. First, we exclude all the large vertices from the graph. Then for the "good" vertices found in the previous step, we also exclude them from $V_{1}$.

Now all vertices have degree at most $t$. In each of $V_{1}, \ldots, V_{k}$, we pack their vertices into groups where in each group the total degree of vertices is at most $t$. Then the total number of groups is bounded by $O(m / t)$.

For each $k$-tuple of groups $\left(G_{1}, \ldots, G_{k}\right)$, where $G_{1} \subseteq V_{1}, \ldots, G_{k} \subseteq V_{k}$, we query the oracle deciding $M C_{\left(\exists x_{1}\right) \varphi^{\prime}}$ whether it accepts on the subgraph induced by vertices in $G_{1}, \ldots, G_{k}$. If so, then we find a vertex $v_{1}$ in $V_{1}$ so that when $x_{1} \leftarrow v_{1}$, the current subgraph satisfies $\varphi^{\prime}$. We remove this $v_{1}$ from $V_{1}$. Then we repeat this process to find new satisfying $v_{1}$ 's in $V_{1}$, and remove these $v_{1}$ 's from $V_{1}$. When $V_{1}$ is empty, or when no new solution is found after all group combinations are exhausted, the algorithm terminates. If in the end $V_{1}$ is empty, then all $v_{1} \in V_{1}$ are in solutions of $M C_{\exists x_{1} \varphi^{\prime}}$, so we accept. Otherwise, we reject.

Each query to $M C_{\exists x_{1} \varphi^{\prime}}$ has size $m^{\prime}=O(k t)=O(t)$. Because the number of different $k$-tuples of groups is $O(m / t)^{k}=O\left((m / t)^{k}\right)$, the number of queries made is $O\left((m / t)^{k}+\left|V_{1}\right|\right) \cdot O(\log m)=$ $O\left(\left(m^{1 / k}\right)^{k}+\left|V_{1}\right|\right) \cdot O(\log m)=O(m \log m)$ times. If $M C_{\exists x_{1} \varphi^{\prime}}$ on input size $m^{\prime}$ is solvable in time $O\left(m^{\prime k} / s\left(m^{\prime}\right)\right)$, then the running time for $M C_{\forall x_{1} \varphi^{\prime}}$ is $O(m \log m) \cdot O\left(m^{\prime k} / s\left(m^{\prime}\right)\right)=O(m \log m$. $\left(m^{(k-1) / k}\right)^{k} / s\left(m^{(k-1) / k}\right) \leq O\left(m^{k} / s(\sqrt{m}) \cdot \log m\right)$. The exponent of $m$ is less than $k$. Thus this is a fine-grained Turing reduction. Lemma 8.1 follows.

Note that this reduction works not only on graphs but also on structures with relations of arity greater than two.

\section{BASELINE AND IMPROVED ALGORITHMS}

In this section, we first present a baseline algorithm for $M C(k+1)$ that runs in time $O\left(n^{k-1} m\right)$, which also implicitly gives us a quantifier-eliminating downward reduction from any $M C(k+1)$ 
problem to $M C(k)$ problems for $k \geq 2$. Then, we show how to get an improved algorithm in time $m^{k} / 2^{\Theta(\sqrt{\log m})}$ using our reductions and the result in References [3, 16]. Finally, we present the algorithms for some specific quantifier structures in $O\left(\mathrm{~m}^{3 / 2}\right)$, so that these problems are easy cases in first-order property problems.

\subsection{Baseline Algorithm for First-order Properties}

This section gives an $O\left(n^{k-1} m\right)$ time algorithm solving $M C(k+1)$ with any quantifier structure for $k \geq 1$, thus proving Lemma 9.1.

LEMMA 9.1 (QUANTIFIER-ELIMINATING DOWNWARD REDUCTION FOR $M C(k+1)$ ). Let the running time of $M C(k+1)$ on graphs of $n$ vertices and $m$ edges be $T_{k}(n, m)$. We have the recurrence

$$
\begin{aligned}
& T_{k}(n, m) \leq n \cdot T_{k-1}(n, O(m))+O(m), \text { for } k \geq 2 . \\
& T_{1}(n, m)=O(m) .
\end{aligned}
$$

By this lemma, if all problems in $M C(k)$ have algorithms in time $T(n, m)$, then any problem in $M C(k+1)$ can be solved in time $n \cdot T(n, m)$.

Base Case. We prove that when $k=1, T_{k}(n, m)=m$. For each $v_{1} \in V_{1}$, the algorithm computes $\#\left(v_{1}\right)=\left|\left\{v_{2} \in V_{2}\left|\left(v_{1}, v_{2}\right)\right|=\psi\right\}\right|$. Thus we can list the sets of $v_{1}$ s.t. \# $\left(v_{1}\right)>0$ (if the inner quantifier is $\exists$ ), or those that satisfy $\#\left(v_{1}\right)=\left|V_{2}\right|$ (if it is $\forall$ ).

Let there be $p_{1}$ different unary predicates on $v_{1}$ and $p_{2}$ different unary predicates on $v_{2}$. We partition the universes $V_{1}$ and $V_{2}$, respectively, into $2^{p_{1}}$ and $2^{p_{2}}$ subsets, based on the truth values of all the unary predicates of the corresponding variable. The number of different pairs of subsets is a constant. Each time, we pick a pair consisting of one subset from $V_{1}$ and one subset from $V_{2}$, and replace the unary predicates by constants. In this way, we can just consider binary predicates in the following argument.

Let $\bar{\psi}\left(v_{1}, v_{2}\right)$ be the formula where each occurrence of each negated binary relation $R_{i}\left(v_{1}, v_{2}\right)$ is replaced by false. We enumerate all tuples $\left(v_{1}, v_{2}\right)$ connected by at least one edge. For each tuple, we evaluate $\psi\left(v_{1}, v_{2}\right)$ and $\bar{\psi}\left(v_{1}, v_{2}\right)$. Let

$$
\# \psi\left(v_{1}\right)=\sum_{v_{2} \text { adjacent to } v_{1}}\left(\left[\psi\left(v_{1}, v_{2}\right)=\text { true }\right]-\left[\bar{\psi}\left(v_{1}, v_{2}\right)=\text { true }\right]\right)
$$

(in which the brackets are Iverson brackets). It can be computed by enumerating all tuples $\left(v_{1}, v_{2}\right)$ connected by at least one edge. Next, because in $\bar{\psi}$ there are no occurrences of negated binary predicates, we can compute

$$
\# \bar{\psi}\left(v_{1}\right)=\text { The number of } v_{2} \text { s.t. } \bar{\psi}\left(v_{1}, v_{2}\right) \text { holds }
$$

by first enumerating all tuples $\left(v_{1}, v_{2}\right)$ connected by at least one edge and checking if $\bar{\psi}\left(v_{1}, v_{2}\right)$ holds, and then considering the number of non-neighboring $v_{2}$ 's for each $v_{1}$, if being a nonneighbor of $v_{1}$ also makes $\bar{\psi}\left(v_{1}, v_{2}\right)$ true. Finally, let $\#\left(v_{1}\right)=\# \psi\left(v_{1}\right)+\# \bar{\psi}\left(v_{1}\right)$.

This algorithm is correct, because whenever a pair $\left(v_{1}, v_{2}\right)$ satisfies $\psi\left(v_{1}, v_{2}\right)$, there are two cases. The first is that there exists an edge between $v_{1}$ and $v_{2}$. In this case, when we enumerate all edges, $\left[\psi\left(v_{1}, v_{2}\right)=\right.$ true $]$ equals one and $\left[\bar{\psi}\left(v_{1}, v_{2}\right)=\right.$ true $]$ equals its contribution to $\# \bar{\psi}\left(v_{1}\right)$. However, if there does not exist an edge between $v_{1}$ and $v_{2}$, then the contribution of $\left(v_{1}, v_{2}\right)$ to \# $\psi\left(v_{1}\right)$ is 0 and to $\# \bar{\psi}\left(v_{1}\right)$ is 1 .

Whenever a pair $\left(v_{1}, v_{2}\right)$ does not satisfy $\psi\left(v_{1}, v_{2}\right)$, there are also two cases. If there exists an edge between $v_{1}$ and $v_{2}$, when we enumerate all edges, then $\left[\psi\left(v_{1}, v_{2}\right)=\right.$ true $]$ equals zero and 
$\left[\bar{\psi}\left(v_{1}, v_{2}\right)=\right.$ true $]$ equals its contribution to $\# \bar{\psi}\left(v_{1}\right)$. However, if there does not exist an edge between $v_{1}$ and $v_{2}$, the contributions of $\left(v_{1}, v_{2}\right)$ to $\# \psi\left(v_{1}\right)$ and to $\# \bar{\psi}\left(v_{1}\right)$ are both 0 .

Inductive Step. For $k \geq 2$, we give a quantifier-eliminating downward reduction, thus proving the recurrence relation. Assume $\varphi=\left(Q_{1} x_{1}\right) \ldots\left(Q_{k+1} x_{k+1}\right) \psi\left(x_{1}, \ldots, x_{k+1}\right)$ For each $v_{1} \in V_{1}$, create new formula $\varphi_{v_{1}}=\left(Q_{2} x_{2}\right) \ldots\left(Q_{k+1} x_{k+1}\right) \psi\left(x_{2}, \ldots, x_{k+1}\right)$, and in $\psi$ we replace each occurrence of unary predicate $R_{i}\left(x_{1}\right)$ with a constant $R_{i}\left(v_{1}\right)$, and replace each occurrence of binary predicate $R_{i}\left(x_{1}, x_{j}\right)$ (or $R_{i}\left(x_{j}, x_{1}\right)$ ) with unary predicate $R_{i}^{\prime}\left(x_{j}\right)$ whose value equals $R_{i}\left(v_{1}, x_{j}\right)$ (or $R_{i}\left(x_{j}, v_{1}\right)$ ), and so on. Our algorithm enumerates all $v_{1} \in V_{1}$, and then computes if the graph induced by $V_{2}, \ldots, V_{k+1}$ satisfies $\varphi_{v_{1}}$. If $x_{1}$ is quantified by $\exists$, then we accept iff any of them accepts. Otherwise, we accept iff all of them accept. The construction of $\varphi_{v_{1}}$ takes time $O(m)$. The created graph has $O(n)$ vertices and $O(m)$ edges. Thus the recursion follows.

This process is a quantifier-eliminating downward reduction from an $M C(k+1)$ problem to an $M C(k)$ problem. It makes $O(m)$ queries, each of size $O(m)$. Then if problems in $M C(k)$ are solvable in time $O\left(m^{k-1-\epsilon}\right)$, then problems in $M C(k+1)$ are solvable in time $m \cdot O\left(m^{k-1-\epsilon}\right)=$ $O\left(m^{k-\epsilon}\right)$. This quantifier-eliminating downward reduction implies that if all $M C(k)$ have $T(n, m)$ time algorithms, then all $M C(k+1)$ problems have $n \cdot T(n, m)$ time algorithms.

From the recursion and the base case, we have the running time $O\left(n^{k-1} m\right)$ by induction. The quantifier-eliminating downward reduction from $M C(k+1)$ to $M C(3)$ in Lemma 9.1 also works for hypergraphs. We exhaustively search the first $k-2$ quantified variables, and by replacing the occurrences of these variables by constants in the formula, we can reduce the arities of relations. After the reduction, we get a hypergraph of max arity at most three.

\subsection{Algorithms for Easy Cases}

In this section, we show that any $(k+1)$-quantifier problem with a quantifier sequence ending with $\exists \exists$ or $\forall \forall$ is solvable in time $O\left(m^{k-1 / 2}\right)$. First, we use the quantifier-eliminating downward reduction to reduce the problem to a $M C$ (3) problem. Then from the next subsections, we see that these problems are solvable in $O\left(\mathrm{~m}^{3 / 2}\right)$. Reference [35] shows improved algorithms that run in time $O\left(\mathrm{~m}^{1.41}\right)$ for detecting triangles and detecting induced paths of length 2, which are special cases of $M C(\exists \exists \exists)$.

Lemma 9.2. Problems in $M C(\exists \exists \exists), M C(\forall \forall \forall), M C(\forall \exists \exists)$, and $M C(\exists \forall \forall)$ are solvable in $O\left(m^{3 / 2}\right)$.

In the first two subsections, we consider when the input structures are graphs. Then in the last subsection, we consider the cases when the input structures have higher arity relations.

9.2.1 Problems in $M C(\exists \exists \exists)$ and $M C(\forall \forall \forall)$. For problems in $M C(\forall \forall \forall)$, we decide its negation, which is a $M C(\exists \exists \exists)$ problem.

We define nine Atomic Problems, which are special MC(3) problems. Let the Atomic Problem labeled by $\ell$ to be $M C_{(\exists x \in X)(\exists y \in Y)(\exists z \in Z) \psi_{\ell}}$, and referred to as $\Delta[\ell]$. It is defined on a tripartite graph on vertex sets $(X, Y, Z)$, whose edge sets are $E_{X Y}, E_{Y Z}, E_{X Z}$ defined on $(X, Y),(Y, Z),(X, Z)$, respectively. The graph is undirected, i.e., $E_{X Y}, E_{Y Z}$, and $E_{X Z}$ are symmetric relations. For simplicity, we define an edge predicate $E$ so that $E\left(v_{1}, v_{2}\right)$ is true iff there is an edge in any of $E_{X Y}, E_{Y Z}, E_{X Z}$ connecting $\left(v_{1}, v_{2}\right)$ or $\left(v_{2}, v_{1}\right)$. Besides, we use $\operatorname{deg}_{Y}(x)$ to denote the number of $x$ 's neighbors in $Y$.

The $\psi_{\ell}$ for all Atomic Problems are defined in Table 1. For problem $M C_{\varphi}$, where $\varphi=(\exists x \in X)$ $(\exists y \in Y)(\exists z \in Z) \psi(x, y, z)$, we write $\psi$ as a DNF, and split the terms. Then we decide if there is a term so that there exist $x, y, z$ satisfying this term. On each term $t$, which is a conjunction of predicates and negated predicates, we work on the induced subgraph whose vertices satisfy all the positive unary predicates and falsify all the negated unary predicates defined on them in $t$. Then we can remove all unary predicates from the conjunction, which is now a conjunction 
Table 1. Atomic Problems

\begin{tabular}{|l|c|c|}
\hline$\psi_{2}=E(x, y) \wedge E(x, z)$ & $\psi_{2+}=E(x, y) \wedge E(x, z) \wedge E(y, z)$ & $\psi_{2-}=E(x, y) \wedge E(x, z) \wedge \neg E(y, z)$ \\
\hline$\psi_{1}=E(x, y) \wedge \neg E(x, z)$ & $\psi_{1+}=E(x, y) \wedge \neg E(x, z) \wedge E(y, z)$ & $\psi_{1_{-}}=E(x, y) \wedge \neg E(x, z) \wedge \neg E(y, z)$ \\
\hline$\psi_{0}=\neg E(x, y) \wedge \neg E(x, z)$ & $\psi_{0+}=\neg E(x, y) \wedge \neg E(x, z) \wedge E(y, z)$ & $\psi_{0-}=\neg E(x, y) \wedge \neg E(x, z) \wedge \neg E(y, z)$ \\
\hline
\end{tabular}

of binary predicates or their negations. (If the conjunction is a single predicate or a single negated predicate, then we can deal with it easily, so we do nt consider this case here.) If we define $E(x, y)=\bigwedge_{R}$ is a positive binary predicate in $t R(x, y) \wedge \bigwedge_{R}$ is a negative binary predicate in $t \neg R(x, y)$, and define $E(y, z)$ and $E(x, z)$ similarly, then $t$ becomes equivalent with some Atomic Problem, or a disjunction of Atomic Problems (because variables $y$ and $z$ are interchangeable, the Atomic Problems and their disjunctions cover all possible cases).

In our algorithm for each problem $\Delta[\ell]$, instead of deciding the existence of satisfying $x, y, z$, we consider these problems as counting problems, where for each $x$ we compute

$$
\#_{\ell}(x)=\mid\left\{(y, z) \mid x, y, z \text { satisfy } \psi_{\ell}\right\} \mid .
$$

Problems $\Delta[2], \Delta[1], \Delta[0]$ can be computed straightforwardly.

- In $\Delta[2], \#_{2}(x)=\operatorname{deg}_{Y}(x) \times \operatorname{deg}_{Z}(x)$.

- In $\Delta[1], \#_{1}(x)=\operatorname{deg}_{Y}(x) \times\left(|Z|-\operatorname{deg}_{Z}(x)\right)$.

- In $\Delta[0], \#_{0}(x)=\left(|Y|-\operatorname{deg}_{Y}(x)\right) \times\left(|Z|-\operatorname{deg}_{Z}(x)\right)$.

Next we show for labels $\ell \in\{2+, 1+, 0+, 2-, 1-, 0-\}$, problems $\Delta[\ell]$ can be computed in $O\left(m^{3 / 2}\right)$. Algorithm 1 solves $\Delta[2+]$, that is, for each $x$, counting the number of triangles that contain $x$. The first part of the algorithm only considers small degree $y$. On each iteration of the outer loop, the inner loop is run for at most $\sqrt{m}$ times. The second part only considers large degree $y$. Because there are at most $\sqrt{m}$ of them, the outer loop is run for at most $\sqrt{m}$ times. Therefore the running time of the algorithm is $O\left(\mathrm{~m}^{3 / 2}\right)$.

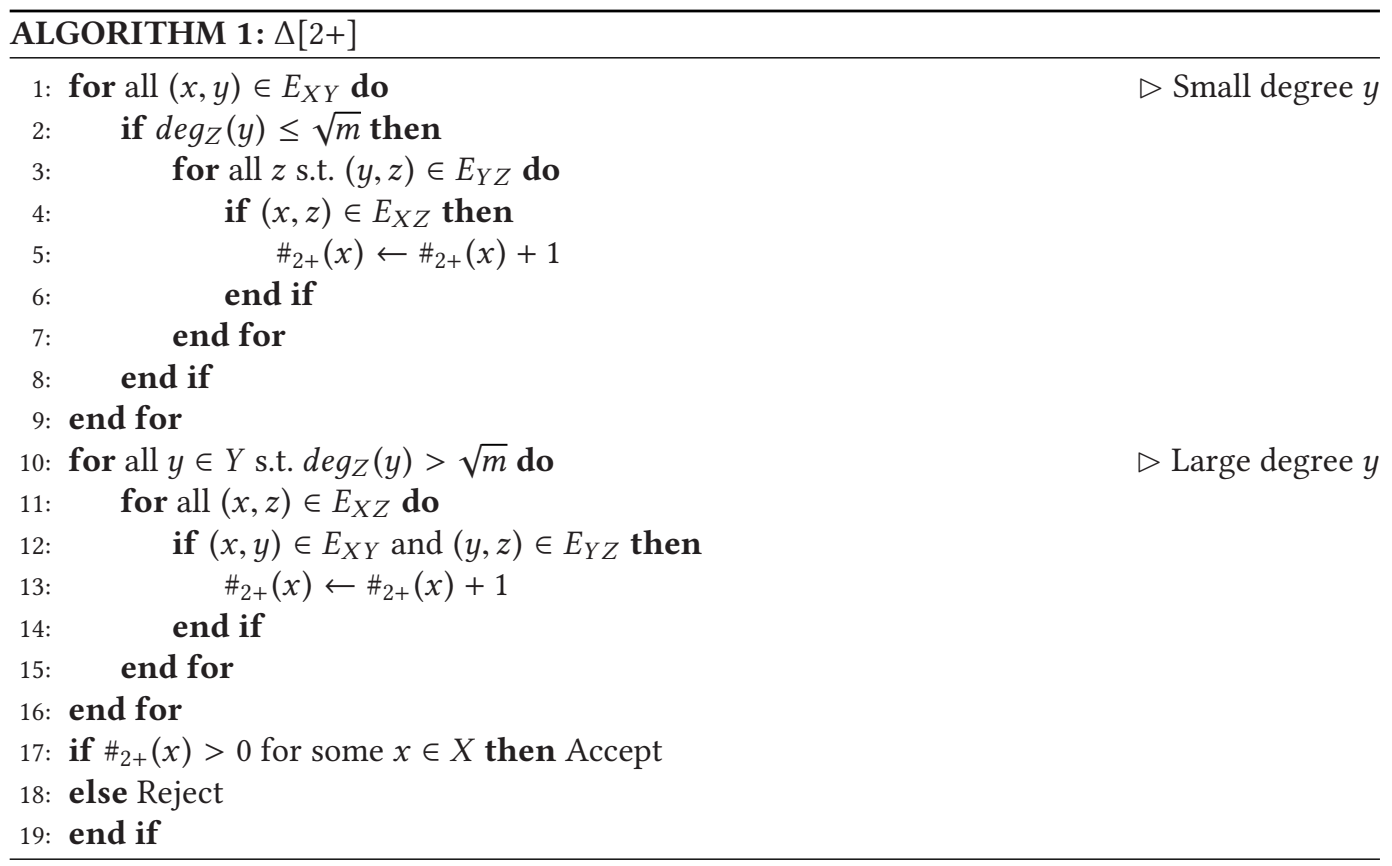


Algorithm 2 solves $\Delta[1+]$, which for each $x$ counts $(x-y-z)$ paths where there is no edge between $x$ and $z$. The first part is similar as $\Delta[2+]$. The second part first over-counts $(x-y-z)$ paths for all large degree $y$ without restricting the edge between $x$ and $z$, and then counts the number of over-counted cases to exclude them from the final result. In the first block, the inner loop is run for at most $\sqrt{m}$ times for each edge in $E_{X Y}$. The second block takes time $O(m)$. The outer loop of the third block is run for at most $\sqrt{m}$ times, because there are at most $\sqrt{m}$ sets with degree at least $\sqrt{m}$. So in all, the running time is $O\left(\mathrm{~m}^{3 / 2}\right)$.

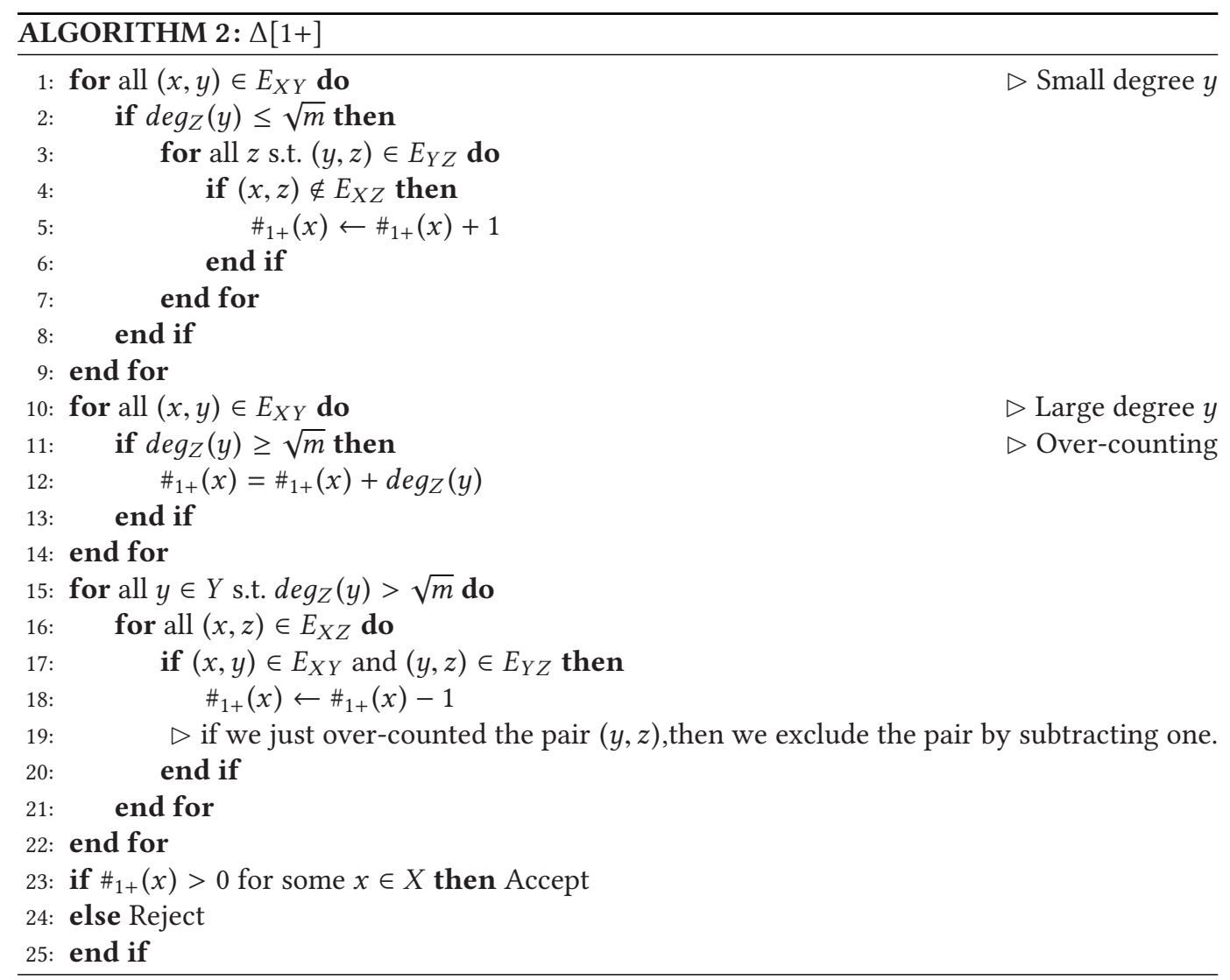

For $\Delta[0+]$, we first compute $\#_{2+}(x)$, which is the result of $\Delta[2+]$, and then compute $\#_{1+}(x)$ and $\#_{1+}^{\prime}(x)$, which are results of $\Delta[1+]$ on vertex sets $(X, Y, Z)$ and $(X, Z, Y)$, respectively. Finally, let $\#_{0+}(x) \leftarrow\left|E_{Y Z}\right|-\left(\#_{2+}(x)+\#_{1+}(x)+\#_{1+}^{\prime}(x)\right)$.

$\#_{2-}(x), \#_{1-}(x), \#_{0-}(x)$ can be computed by respectively taking the differences of $\#_{2}(x), \#_{1}(x), \#_{0}(x)$ and $\#_{2+}(x), \#_{1+}(x), \#_{0+}(x)$.

9.2.2 Problems in $M C(\forall \exists \exists)$ and $M C(\exists \forall \forall)$. For problems in $M C(\exists \forall \forall)$, we decide its negation, which is a $M C(\forall \exists \exists)$ problem.

For problem $M C_{\varphi}$, where $\varphi=(\forall x \in X)(\exists y \in Y)(\exists z \in Z) \psi(x, y, z)$, we use the same algorithm to compute $\#_{\ell}(x)$ for all $x \in X$. If the value of $\#_{\ell}(x)$ is greater than zero for all $x \in X$, then we accept, otherwise reject. Again, we write $\psi$ as a DNF, and split the terms. By the same argument as the previous lemma, we transform the problem to a disjunction of Atomic Problems. If for all 
$x \in X$, at least in one of the Atomic Problem, $\#_{\ell}(x)$ is greater than zero, then we accept; otherwise, reject.

9.2.3 Structures with Higher Arity Relations. The above algorithms can be extended to structures with relations of arity greater than two. First, we write the quantifier-free part $\psi$ in DNF and split each term to a separate $\exists \exists \exists$ problem (or $\forall \exists \exists$, respectively). Then for each term $\psi_{t}$, we decide if there exist $x_{1}, x_{2}, x_{3}$ satisfying it. Let $\psi_{t 1}$ be the part of the conjunction containing all ternary predicates in $\psi_{t}$, and $\psi_{t 2}$ be the rest of term $\psi_{t}$. Thus $\psi_{t}=\psi_{t 1} \wedge \psi_{t 2}$.

If in $\psi_{t}$ some ternary predicate occurs positively, then we can just count $\#\left(x_{1}\right)$ on the subgraph where $\psi_{t 1}$ is true.

If all ternary predicates in $\psi_{t}$ occur negatively, then we first count \# $\left(x_{1}\right)$ satisfying formula $\psi_{t 2}$, and then we count $\#^{\prime}\left(x_{1}\right)$ on the subgraph where $\psi_{t 1}$ is true. Finally, we subtract $\#^{\prime}\left(x_{1}\right)$ from $\#\left(x_{1}\right)$ for each $x_{1}$.

If $\psi_{t}$ has no ternary relations, then we just count $\#\left(x_{1}\right)$ using the algorithm for graphs.

\section{IMPROVED ALGORITHMS}

In this section, we present an algorithm solving Sparse OV in time $m^{2} / 2^{\Theta(\sqrt{\log m})}$. It is based on the articles $[3,16]$, which solves dense OV for vectors of dimension $d$ in time $n^{2-\Omega(1 / \log (d / \log n))}$.

Consider the universe-shrinking self-reduction for Sparse OV (Set Disjointness) in Section 6. We show that for $s(m)=2^{\Theta(\sqrt{\log m})}$, by the above theorem, this reduction gives an algorithm in time $m^{2} / 2^{\Theta(\sqrt{\log m})}$. We deal with large sets and small sets separately. For sets of size at least $s(m)$, we check if each of them is disjoint with some other set. From the argument for large sets, this is in time $\mathrm{m}^{2} / \mathrm{s}(\mathrm{m})$. Then, for sets of size less than $s(m)$, we use the universe-shrinking selfreduction to reduce this instance to a Sparse OV instance on universe of size $s(m)^{\frac{5}{6 k}}$ (in which case $k=2)$. Using the algorithm from References $[3,16]$, we can solve it in time $n^{2-\Theta\left(1 / \log \left(s(m)^{\frac{5}{6 k}}\right)\right)} \leq$ $m^{2-\Theta(1 / \log (s(m))} \leq m^{2} / 2^{\Theta(\log m / \log s(m))}=m^{2} / 2^{\Theta(\sqrt{\log m})}$. So the total running time is bounded by $m^{2} / 2^{\Theta(\sqrt{\log m})}$.

By the above argument and Theorem 1.2, since all the Basic Problems are solvable in time $m^{2} / 2^{\Theta(\sqrt{\log m})}$, so is any other problem in $M C(\exists \exists \forall)$. The reduction from $M C(\forall \exists \forall)$ to $M C(\exists \exists \forall)$ in Section 8 gives $2^{\Theta(\sqrt{\log m})}$ savings for $M C(\forall \exists \forall)$ problems. Reducing to three-quantifier case by brute-forcing over the first $k-2$ variables we get Theorem 1.4, that states all $M C(k+1)$ problems can be solved in $m^{k} / 2^{\Theta(\sqrt{\log m})}$ time.

\section{OPEN PROBLEMS}

An obvious open problem is whether a similar kind of equivalence exists for the dense case of OV. Is it "fine-grained equivalent" to some natural complexity class?

Our results raise the possibility that many other classes have complete problems under finegrained reducibility, and that this will be a general method for establishing the plausibility of conjectures on the fine-grained complexity of problems. There is a number of candidates for such classes. We could drop the restriction that the formula has $k$ quantifiers in all, and look at formulas with quantifier depth $k .{ }^{9}$ We could also stratify the first-order formulas by variable complexity, the number of distinct variable names in a formula, rather than number of quantifiers. (Variable complexity arises naturally in database theory, because the variable complexity determines the arity of some relation in any way of expressing the query as a sequence of sub-queries.) First-order

\footnotetext{
${ }^{9}$ For example, $\exists(x)\left(\exists y\left(\exists z \psi_{1}(x, y, z) \wedge \forall z \psi_{2}(x, y, z)\right) \wedge \forall y\left(\exists z \psi_{3}(x, y, z) \vee \forall z \psi_{4}(x, y, z)\right)\right)$ has quantifier depth 3.
} 
logic is rather limited, so we could look at augmentations that increase its reach, such as allowing a total ordering on elements, or allowing the logic to take transitive closures of relations (e.g., to talk about the reachability relation in a sparse directed graph), or more generally, introduce monotone fixed point operations. Alternatively, rather than varying the types of formulas, we could restrict the types of structures, for example, considering structures of bounded treewidth.

It would be interesting to find more reductions between and equivalences among the problems that are proven hard under some conjecture. For example, Edit Distance, Fréchet Distance, and Longest Common Subsequence are all almost quadratically hard assuming SETH. Are there any reductions between these problems? Are they all equivalent as far as having subquadratic algorithms? All of these problems have similar dynamic programming formulations. Can we formalize a class of problems with such dynamic programming algorithms and find complete problems for this class? More generally, we would like taxonomies of the problems within $\mathrm{P}$ that would classify more of the problems that have conjectured hardness, or have provable hardness based on conjectures about other problems. Such a taxonomy might have to be based on the structure of the conjectured best algorithms for the problems rather than on resource limitations.

\section{ACKNOWLEDGMENTS}

First, we thank Virginia Vassilevska Williams for her inspiring ideas. We thank Marco Carmosino, Anant Dhayal, Ivan Mihajlin, and Victor Vianu for proofreading and suggestions on this article. We also thank Valentine Kabanets, Ramamohan Paturi, Ramyaa Ramyaa, and Stefan Schneider for many useful discussions. Finally, we really appreciate the suggestions from the referees about the writing and expression.

\section{REFERENCES}

[1] Amir Abboud, Arturs Backurs, and Virginia Vassilevska Williams. 2015. Quadratic-time hardness of LCS and other sequence similarity measures. CoRR abs/1501.07053 (2015).

[2] Amir Abboud, Thomas Dueholm Hansen, Virginia Vassilevska Williams, and Ryan Williams. 2016. Simulating branching programs with edit distance and friends: Or: A polylog shaved is a lower bound made. In Proceedings of the STOC. ACM, 375-388.

[3] Amir Abboud, Ryan Williams, and Huacheng Yu. 2015. More applications of the polynomial method to algorithm design. In Proceedings of the 26th Annual ACM-SIAM Symposium on Discrete Algorithms. SIAM, 218-230.

[4] Amir Abboud, Virginia Vassilevska Williams, and Joshua Wang. 2016. Approximation and fixed parameter subquadratic algorithms for radius and diameter in sparse graphs. In Proceedings of the 27th Annual ACM-SIAM Symposium on Discrete Algorithms. SIAM, 377-391.

[5] Amir Abboud, Virginia Vassilevska Williams, and Oren Weimann. 2014. Consequences of faster alignment of sequences. In Automata, Languages, and Programming. Springer, 39-51.

[6] Miklos Ajtai and Avi Wigderson. 1985. Deterministic simulation of probabilistic constant depth circuits. In Proceedings of the 26th Annual Symposium on Foundations of Computer Science 1985. IEEE, 11-19.

[7] Noga Alon, Raphael Yuster, and Uri Zwick. 1995. Color-coding. J. ACM 42, 4 (1995), 844-856.

[8] László Babai, Lance Fortnow, Noam Nisan, and Avi Wigderson. 1993. BPP has subexponential time simulations unless EXPTIME has publishable proofs. Comput. Complex. 3 (1993), 307-318.

[9] Arturs Backurs and Piotr Indyk. 2015. Edit distance cannot be computed in strongly subquadratic time (unless SETH is false). In Proceedings of the 47th Annual ACM on Symposium on Theory of Computing (STOC'15). 51-58. DOI : https:// doi.org/10.1145/2746539.2746612

[10] David Mix Barrington, Neil Immerman, and Howard Straubing. 1990. On uniformity within NC ${ }^{1}$. fournal of Computer and System Sciences 41, 3 (1990), 274-306.

[11] Michele Borassi, Pierluigi Crescenzi, and Michel Habib. 2016. Into the square: On the complexity of some quadratictime solvable problems. Electr. Notes Theor. Comput. Sci. 322 (2016), 51-67.

[12] Karl Bringmann. 2014. Why walking the dog takes time: Fréchet distance has no strongly subquadratic algorithms unless SETH fails. In Proceedings of the 2014 IEEE 55th Annual Symposium on Foundations of Computer Science (FOCS'14). IEEE, 661-670. 
[13] Karl Bringmann and Marvin Kunnemann. 2015. Quadratic conditional lower bounds for string problems and dynamic time warping. In Proceedings of the 2015 IEEE 56th Annual Symposium on Foundations of Computer Science (FOCS'15). IEEE, 79-97.

[14] Marco L. Carmosino, Jiawei Gao, Russell Impagliazzo, Ivan Mihajlin, Ramamohan Paturi, and Stefan Schneider. 2016. Nondeterministic extensions of the strong exponential time hypothesis and consequences for non-reducibility. In Proceedings of the 2016 ACM Conference on Innovations in Theoretical Computer Science. ACM, 261-270.

[15] Marco L. Carmosino, Russell Impagliazzo, Valentine Kabanets, and Antonina Kolokolova. 2016. Learning algorithms from natural proofs. In Proceedings of the 31st Conference on Computational Complexity.

[16] Timothy M. Chan and Ryan Williams. 2016. Deterministic APSP, orthogonal vectors, and more: Quickly derandomizing Razborov-Smolensky. In Proceedings of the 27th Annual ACM-SIAM Symposium on Discrete Algorithms. SIAM, $1246-1255$.

[17] Moses Charikar, Piotr Indyk, and Rina Panigrahy. 2002. New algorithms for subset query, partial match, orthogonal range searching, and related problems. In Automata, Languages and Programming. Springer, 451-462.

[18] Rodney G. Downey and Michael R. Fellows. 1992. Fixed-parameter intractability. In Proceedings of the 7th Annual Structure in Complexity Theory Conference 1992. IEEE, 36-49.

[19] Jörg Flum and Martin Grohe. 2006. Parameterized Complexity Theory, volume XIV of Texts in Theoretical Computer Science.

[20] Lester R. Ford Jr. 1956. Network Flow Theory. Technical Report. DTIC Document.

[21] Russell Impagliazzo, Valentine Kabanets, and Avi Wigderson. 2002. In search of an easy witness: Exponential versus probabilistic time. f. Comput. System Sci. 65, 69 (2002), 672-694.

[22] Russell Impagliazzo and Ramamohan Paturi. 1999. Complexity of $k$-SAT. In Proceedings of the 14th Annual IEEE Conference on Computational Complexity 1999. IEEE, 237-240.

[23] Russell Impagliazzo, Ramamohan Paturi, and Francis Zane. 1998. Which problems have strongly exponential complexity? In Proceedings of the 39th Annual Symposium on Foundations of Computer Science 1998. IEEE, 653-662.

[24] David S. Johnson and Mario Szegedy. 1999. What are the least tractable instances of max independent set? In Proceedings of the 10th Annual ACM-SIAM Symposium on Discrete Algorithms. Society for Industrial and Applied Mathematics, 927-928.

[25] Valentine Kabanets and Russell Impagliazzo. 2004. Derandomizing polynomial identity tests means proving circuit lower bounds. Comput. Complex. 13, 1-2 (2004), 1-46.

[26] Nathan Linial, Yishay Mansour, and Noam Nisan. 1993. Constant depth circuits, Fourier transform, and learnability. f. ACM 40, 3 (1993), 607-620. DOI : https://doi.org/10.1145/174130.174138

[27] Daniel Moeller, Ramamohan Paturi, and Stefan Schneider. 2016. Subquadratic algorithms for succinct stable matching. In Proceedings of the International Computer Science Symposium. Springer, 294-308.

[28] Noam Nisan and Avi Wigderson. 1994. Hardness vs. randomness. f. Comput. Syst. Sci. 49 (1994), 149-167.

[29] Richard Edwin Stearns and Harry B. Hunt III. 1990. Power indices and easier hard problems. Math. Syst. Theory 23, 1 (1990), 209-225.

[30] Moshe Y. Vardi. 1982. The complexity of relational query languages. In Proceedings of the 14th Annual ACM Symposium on Theory of Computing. ACM, 137-146.

[31] Ryan Williams. 2005. A new algorithm for optimal 2-constraint satisfaction and its implications. Theor. Comput. Sci. 348, 2 (2005), 357-365.

[32] Ryan Williams. 2013. Improving exhaustive search implies superpolynomial lower bounds. SIAM f. Comput. 42,3 (2013), 1218-1244.

[33] Ryan Williams. 2014. Faster decision of first-order graph properties. In Proceedings of the foint Meeting of the 23rd EACSL Annual Conference on Computer Science Logic (CSL) and the 29th Annual ACM/IEEE Symposium on Logic in Computer Science (LICS). ACM, 80.

[34] Ryan Williams. 2014. Nonuniform ACC circuit lower bounds. f. ACM 61, 1 (2014), 2:1-2:32. DOI : https://doi.org/10. $1145 / 2559903$

[35] Virginia Vassilevska Williams. 2016. CS267 Lecture 1, Algorithms for Fixed Subgraph Isomorphism. http://theory. stanford.edu/ virgi/cs267/lecture1.pdf.

[36] Virginia Vassilevska Williams and Ryan Williams. 2010. Subcubic equivalences between path, matrix and triangle problems. In Proceedings of the 2010 51st Annual IEEE Symposium on Foundations of Computer Science (FOCS'10). IEEE, 645-654.

[37] Andrew C Yao. 1982. Theory and applications of trapdoor functions. In Proceedings of the Annual IEEE Symposium on Foundations of Computer Science (FOCS'82). 80-91.

Received February 2017; revised March 2018; accepted March 2018 